\title{
In-situ XRD study of actuation mechanisms in BiFeO3- K0.5Bi0.5TiO3-PbTiO3 ceramics
}

DOI:

10.1016/j.actamat.2019.02.026

Document Version

Accepted author manuscript

Link to publication record in Manchester Research Explorer

\section{Citation for published version (APA):}

Li, Y., Chen, Y., Zhang, Z., Kleppe, A., \& Hall, D. (2019). In-situ XRD study of actuation mechanisms in BiFeO3K0.5Bi0.5TiO3-PbTiO3 ceramics. Acta Materialia, 168, 411-425. https://doi.org/10.1016/j.actamat.2019.02.026

\section{Published in:}

Acta Materialia

\section{Citing this paper}

Please note that where the full-text provided on Manchester Research Explorer is the Author Accepted Manuscript or Proof version this may differ from the final Published version. If citing, it is advised that you check and use the publisher's definitive version.

\section{General rights}

Copyright and moral rights for the publications made accessible in the Research Explorer are retained by the authors and/or other copyright owners and it is a condition of accessing publications that users recognise and abide by the legal requirements associated with these rights.

\section{Takedown policy}

If you believe that this document breaches copyright please refer to the University of Manchester's Takedown Procedures [http://man.ac.uk/04Y6Bo] or contact uml.scholarlycommunications@manchester.ac.uk providing relevant details, so we can investigate your claim.

\section{OPEN ACCESS}




\title{
In-situ XRD study of actuation mechanisms in
}

\section{$\mathrm{BiFeO}_{3}-\mathrm{K}_{0.5} \mathrm{Bi}_{0.5} \mathrm{TiO}_{3}-\mathrm{PbTiO}_{3}$ ceramics}

\author{
Yizhe Li ${ }^{\mathrm{a}}$, Ying Chen ${ }^{\mathrm{a}}$, Zhenbo Zhang ${ }^{\mathrm{a}}$, Annette Kleppe ${ }^{\mathrm{b}}$, David A. Hall ${ }^{\mathrm{a},{ }^{*}}$ \\ a School of Materials, University of Manchester, Oxford Rd, Manchester, M13 9PL, \\ UK \\ b Diamond Light Source Ltd, Harwell Science and Innovation Campus, Didcot OX11 \\ ODE, UK.
}

\begin{abstract}
In the present study, we report a nonergodic relaxor ferroelectric composition for high temperature piezoelectric applications, $0.57 \mathrm{BiFeO}_{3}-0.21 \mathrm{~K}_{0.5} \mathrm{Bi}_{0.5} \mathrm{TiO}_{3^{-}}$ $0.22 \mathrm{PbTiO}_{3}$, which exhibits $\mathrm{T}_{\mathrm{m}}$ around $420^{\circ} \mathrm{C}$. By combining the results of in-situ synchrotron XRD and strain measurements using digital image correlation, a pseudocubic nonergodic relaxor to rhombohedral ferroelectric transformation is identified, accompanied by a volume strain close to zero. A methodology is developed to determine the crystallographic parameters of the transformed rhombohedral ferroelectric phase in a strain-free state, using the invariant intersection for diffraction stress analysis. The phase transformation process was analyzed by methods combining peak profile fitting and full pattern refinement; the results obtained illustrate the strain arising from the phase transformation, together with intrinsic/extrinsic contributions and anisotropy in the field-induced strain. The study reveals unusual microscopic strain behavior, distinguished from that of normal rhombohedral ferroelectrics, showing the combined properties of ergodic and normal ferroelectric materials and leading to a dominant intrinsic lattice strain together with a weaker extrinsic domain switching effect. The elastic coupling between different grain families is also reflected in their similar strain orientation distribution (SOD) functions.
\end{abstract}

Key words: Electroceramics; Piezoelectricity; In-situ X-ray diffraction

${ }^{*}$ Corresponding author.

E-mail address: david.a.hall@manchester.ac.uk 


\section{Introduction}

Non-ergodic (NR) relaxor ferroelectrics exhibit the combined characteristics of relaxor and normal ferroelectrics. The long-range ordered polar state is suppressed by the predominant frozen polar nano regions (PNRs) constrained by random electric and strain fields[1]. Nevertheless, a NR can be irreversibly transformed to a ferroelectric (FE) phase with long-range polar order by the application of an electric field beyond a critical level[2]. The transformation is accompanied by a change of crystal structure, from pseudocubic to a noncentrosymmetric structure, which is typically rhombohedral or tetragonal. Such phase transformations are also usually accompanied by changes in volume. These features yield useful dielectric, ferroelectric and piezoelectric properties, leading to applications in electromechanical sensing and actuation, energy storage and harvesting.

The non-ergodic relaxor ferroelectric state has been reported in many complex oxides with perovskite structure. The most widely studied $\mathrm{Pb}\left(\mathrm{Mg}_{1 / 3} \mathrm{Nb}_{2 / 3}\right) \mathrm{O}_{3}$ relaxor single crystal along $\langle 111\rangle_{\mathrm{cub}}$ exhibits a freezing temperature, $T_{f}$ of $210 \mathrm{~K}$ and a critical electric field strength, $E_{c}$ of $1.75 \mathrm{kV} / \mathrm{cm}[1]$. Similar observations have been reported in lanthanum modified lead zirconate titanate 9/65/35 (PLZT) ceramics with $T_{f}$ around $250 \mathrm{~K}[3]$. The NR behavior was also reported in lead-free $\mathrm{BaTi}_{0.675} \mathrm{Zr}_{0.35} \mathrm{O}_{3}$ and $\mathrm{BaTi}_{0.3} \mathrm{Sn}_{0.3} \mathrm{O}_{3}[4,5]$, with $T_{f}$ around $150 \mathrm{~K}$ and $<100 \mathrm{~K}$ respectively. In recent years, more NRs have been reported with $T_{f}$ higher than room temperature, far beyond the cryogenic temperature range. These include $\mathrm{Na}_{0.5} \mathrm{Bi}_{0.5} \mathrm{TiO}_{3}, \mathrm{Bi}\left(\mathrm{Mg}_{1 / 2} \mathrm{Ti}_{1 / 2}\right) \mathrm{O}_{3}$ and $\mathrm{BiFeO}_{3}$ based materials[6-8], which provide stable properties after poling due to the irreversible NR to normal FE transformation. Hence, such materials can satisfy the requirements for applications both around room temperature and at elevated temperatures exceeding those of conventional lead zirconate titanate (PZT) ceramics $[9,10]$.

With the increasing research interest in NR materials, understanding the relationships between microstructure and macroscopic properties becomes a priority, in order to guide new materials research and development. In-situ X-ray 
powder diffraction offers a powerful tool to detect crystallographic information, such as crystal structure, lattice parameters and texture, under the application of external stimuli (temperature, stress, electric field)[11]. The quantification of lattice strain and crystallographic texture due to ferroelectric domain reorientation has been achieved using several different methods, which have been developed based on the characteristics of normal ferroelectric and relaxor materials[12-14]. However, there are still some problems in effectively quantifying the electric fieldinduced strain by analysis of the measured diffraction patterns, due to the occurrence of elastic and piezoelectric anisotropy. This may lead to the misinterpretation of the measured XRD patterns if suitable physical models are not utilised in the fitting procedures. Therefore, single peak profile fitting and full pattern refinement methods incorporating such models have been proposed in order to address these problems [15-18]. For both of these approaches, the crystallographic parameters corresponding to the unpoled state are required to precisely evaluate the electric field-induced strain.

In poled normal ferroelectric ceramics, a remanent strain, $\varepsilon^{p}$, is always observed after removal of the electric field, even though the piezoelectric strain reduces to zero. This remanent strain is associated with the domain reorientation that is effected by the poling process. If we assume volume-conservative domain switching behaviour and transverse isotropy, the macroscopic remanent strain due to poling along $X_{3}$ can be expressed in the coordinate system, $X$, of the specimen as:

$$
\varepsilon^{p}(X)=\left(\begin{array}{ccc}
-\bar{\varepsilon} / 2 & 0 & 0 \\
0 & -\bar{\varepsilon} / 2 & 0 \\
0 & 0 & \bar{\varepsilon}
\end{array}\right)
$$

where the longitudinal and transverse strains along $\left(\psi=0^{\circ}\right)$ and perpendicular $\left(\psi=90^{\circ}\right)$ to the direction of the applied electric field are $\bar{\varepsilon}$ and $-\bar{\varepsilon} / 2$ respectively[19]. If the coordinate system, $X$, is transformed by rotation about $X_{1}$, the strain, $\varepsilon^{p}$, associated with a certain azimuthal angle, $\psi$, is given as:

$$
\varepsilon^{p}(\psi)=\bar{\varepsilon}\left(\frac{3}{2} \cos ^{2} \psi-\frac{1}{2}\right)
$$


Therefore, the presence of a strain free status along an invariant angle $\psi_{0}$, which satisfies $\cos ^{2} \psi_{0}=\frac{1}{3}$, is valid according to the relationship in Eq. 2 [19]. Furthermore, crystallographic planes with their scattering vectors oriented at $\psi_{0}$ can be considered to represent the reference strain-free state with random domain orientation. This is a particularly useful feature for analysis of the electric fieldinduced FE state in a NR, since the crystallographic parameters of the reference (zero-field) state of the transformed phase are not otherwise available[20].

In a normal ferroelectric polycrystal after uniaxial poling along $X_{3}$, the fieldinduced piezoelectric strain, $\varepsilon^{E}$, due to the combination of ferroelectric domain switching and intrinsic piezoelectricity for an applied electric field $E$ along the poling direction $X_{3}$ is:

$$
\varepsilon^{E}=\left(\begin{array}{ccc}
d_{31} E & 0 & 0 \\
0 & d_{31} E & 0 \\
0 & 0 & d_{33} E
\end{array}\right)
$$

where the piezoelectric behavior is characterized by the longitudinal and transverse strain coefficients, $d_{33}$ and $d_{31}$ respectively. Considering the strain expressed as a function of the azimuthal angle, $\psi$, the projection of the strain along the direction $\mathrm{u}_{\psi}$ is initially zero for all $\psi$. Under electric field, the projection of $\varepsilon^{E}$ along $u_{\psi}$ is a linear function of $\cos ^{2} \psi$, given as[21]:

$$
\varepsilon^{E}(\psi)={ }^{t} \mathbf{u}_{\psi} \cdot \varepsilon^{E} \cdot \mathbf{u}_{\psi}=\left(d_{33}-d_{31}\right) E \cos ^{2} \psi+d_{31} E
$$

The coefficients $d_{33}$ and $d_{31}$ can be measured in terms of the longitudinal and transverse strains at a given electric field, for $\psi=0^{\circ}$ and $\psi=90^{\circ}$ respectively. According to Eq. 4 , the direction of the invariant angle, $\psi_{0}$, along which no piezoelectric strain occurs, is given by:

$$
\cos ^{2} \psi_{0}=-d_{31} /\left(d_{33}-d_{31}\right)
$$

which can be assumed as independent of the amplitude of electric field as long as the linear piezoelectric relations are approximately valid. It is also noteworthy that $d_{31}$ is negative and its magnitude is often approximately half that of $d_{33}$ (i.e. a volume conserving process) in most cases of uniaxially poled piezoelectric 
polycrystals, which also results in $\cos ^{2} \psi_{0} \approx 1 / 3\left(\psi_{0} \approx 54.7^{\circ}\right)$ according to Eq. 5 . On the other hand, $\cos ^{2} \psi_{0}$ may deviate from $1 / 3$ in some circumstances, if the relationship $d_{33} \approx-2 d_{31}$ is no longer true or if there are significant volume variations during the poling process, as shown in Figure 1.

The electric field-induced macroscopic strain of NR materials encompasses a combination of the electric field-induced phase transformation strain, lattice strain (intrinsic) and domain switching (extrinsic). The induced phase transformation strain, $\varepsilon_{V}^{\text {trans }}$, is volumetric or dilatational, which results in an equivalent strain, $\varepsilon_{i i}^{\text {trans }}$, in each respective sample dimension. These two statements are described mathematically by the following equations[15]:

$$
\begin{gathered}
\varepsilon_{i i}^{\text {total }}=\varepsilon_{i i}^{\text {trans }}+\varepsilon_{i i}^{\text {intrinsic }}+\varepsilon_{i i}^{\text {extrinsic }} \\
\varepsilon_{i i}^{\text {trans }}=\frac{\varepsilon_{V}^{\text {trans }}}{3}
\end{gathered}
$$

The aforementioned approximation of volume-conservation will be invalid if the transformation strain is sufficiently high and reversible during the poling process. Therefore, the concept of a constant invariant angle is only suitable for systems that possess limited transformation strain or fully transformed systems with irreversible transformation strain. These principles offer the opportunity to quantify the crystallographic parameters of the electric field-induced FE phase in a NR state by evaluation of the diffraction patterns corresponding to the invariant angle, $\psi_{0}$. 


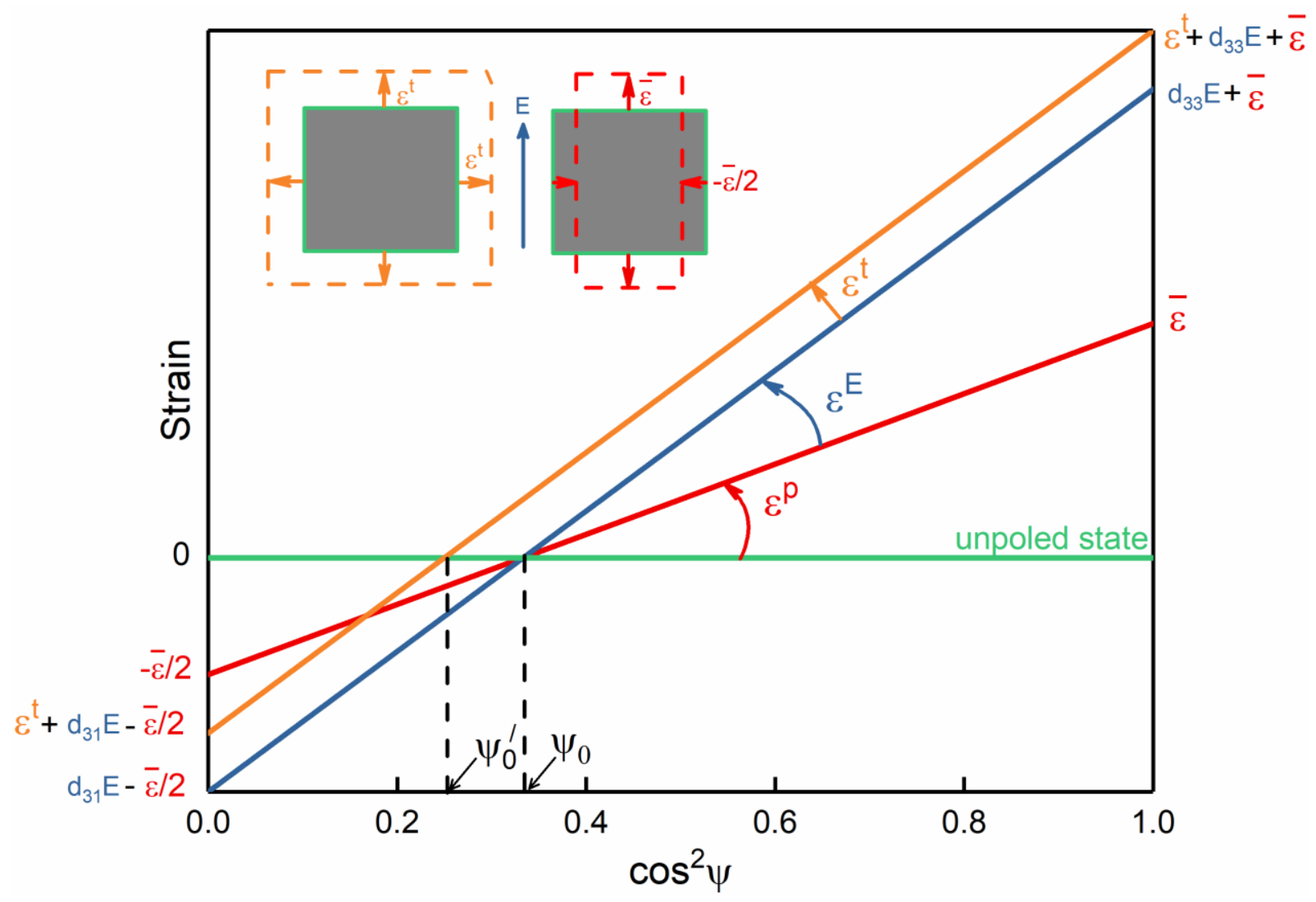

Figure 1. Schematic illustration of azimuthal angle-dependence of remanent poling strain $\left(\varepsilon^{P}\right)$, piezoelectric strain $\left(\varepsilon^{E}\right)$ and the transformation strain $\left(\varepsilon^{t}\right)$ for a poling process beginning from an unpoled NR state. The invariant angles, $\psi_{0}$ and $\psi_{0}^{\prime}$, correspond to cases with and without a linear transformation strain respectively.

The ternary system of $x \mathrm{BiFeO}_{3}-\mathrm{yBi}_{0.5} \mathrm{TiO}_{3^{-}}(1-\mathrm{x}-\mathrm{y}) \mathrm{PbTiO}_{3}(\mathrm{BF}-\mathrm{KBT}-\mathrm{PT})$, which can be used beyond the upper limit of the applicable temperature range for PZT ceramics, has been developed and commercialized for high temperature piezoelectric applications [22]. A relaxor-like behavior for the BF-KBT-PT system has been suggested by temperature-dependent dielectric studies. However, the details of the crystal structure and corresponding actuation mechanisms have not been clarified yet. In the present study, we reveal a NR to FE transformation in the composition $0.57 \mathrm{BiFeO}_{3}-0.21 \mathrm{~K}_{0.5} \mathrm{Bi}_{0.5} \mathrm{TiO}_{3}-0.225 \mathrm{PbTiO}_{3}$, from a pseudocubic $\mathrm{NR}$ phase to rhombohedral FE phase, accompanied by a volume strain close to zero. This specific composition was selected to provide a single-phase BF-KB-PT ceramic that would be suitable for studies of ferroelectric domain switching and actuation mechanisms in this system. The phase transformation process was characterized 
using in-situ synchrotron XRD and analyzed by combining single peak profile fitting and full pattern refinement methods to investigate the strain of the phase transformation, together with intrinsic and extrinsic strain contributions under the application of an electric field, which reveals a somewhat different microscopic strain behavior to that of normal rhombohedral ferroelectrics.

\section{Experimental procedures}

\subsection{Sample preparation}

The $0.57 \mathrm{BiFeO}_{3}-0.21 \mathrm{~K}_{0.5} \mathrm{Bi}_{0.5} \mathrm{TiO}_{3}-0.22 \mathrm{PbTiO}_{3}$ ceramics were prepared by solid state reaction. Initially, the stoichiometric mixture of starting oxide powders was mixed using commercially available powders of $\mathrm{Bi}_{2} \mathrm{O}_{3}(99.9 \%), \mathrm{Fe}_{2} \mathrm{O}_{3}(99 \%), \mathrm{K}_{2} \mathrm{CO}_{3}$ (99.9\%), $\mathrm{PbO}(99.9 \%)$ and $\mathrm{TiO}_{2}(99.9 \%)$ (Sigma-Aldrich). It should also be noted that 0.1 wt\% boric acid $(99.9 \%)$ was added in order to enhance the sintering ability of the $\mathrm{BiFeO}_{3}$-based system. The mixture of starting oxide powders was then milled with yttria-stabilized zirconia beads as milling media in propan-2-ol for 24 hours. Afterwards, the dried powders were calcined at $800^{\circ} \mathrm{C}$ for 4 hours in air with another subsequent 24 hours of milling. The powders were then dried and uniaxially pressed into pellets at a pressure of $50 \mathrm{MPa}$. The ceramic pellets were sintered at $1080^{\circ} \mathrm{C}$ for 2 hours in order to obtain dense samples. Bar-shaped samples were cut from the ceramic pellets into dimensions $10 \mathrm{~mm} \times 1 \mathrm{~mm} \times 1$ $\mathrm{mm}$ with diamond wheel cutting, in order to satisfy the requirements for transmission-mode high energy $\mathrm{X}$-ray diffraction.

\subsection{In-situ high energy X-ray diffraction}

High energy X-ray diffraction (XRD) experiments were performed at Beamline I15, Diamond Light Source, UK with a photon energy of $76 \mathrm{keV}$, a beam diameter of $70 \mu \mathrm{m}$ and using transmission geometry. The XRD data collection was synchronised with an in-situ poling process performed on the ceramic samples; the electric field profile comprises several bipolar cycles with a frequency of around $10 \mathrm{mHz}$ and an amplitude of $5 \mathrm{kV} / \mathrm{mm}$. The sample was immersed in silicone oil in order to avoid electrical breakdown due to the sustained high field at 
such a low frequency. The 2D diffraction patterns were recorded using a PerkinElmer XRD1621 flat-panel detector placed about $1 \mathrm{~m}$ away from the sample, with an acquisition time of $10 \mathrm{~s}$. The calibration and caking procedures for the $2 \mathrm{D}$ diffraction patterns were performed with the software DAWN developed by Diamond Light Source [23]. Calibration was achieved with the measured diffraction pattern of a standard $\mathrm{LaB}_{6}$ powder, before placing the ceramic samples in the holder. 2D diffraction patterns were caked into 24 banks ( $15^{\circ} / \mathrm{bank}$ ) along azimuthal angles, $\psi$, from 0 to $360^{\circ}$ and integrated into 24 corresponding 1-D line profiles. Further details of data collection and post processing are reported in our previous study [24]. Afterwards, the 1-D line profiles were processed with Topas, MAUD and in-house developed MATLAB script in order to obtain the crystallographic parameters by full pattern refinement and single peak profile fitting. In particular, multiple physical functions in MAUD were adopted to accommodate the field-induced texture and anisotropy as reported previously $[16,25]$.

\subsection{Digital image correlation}

In order to duplicate the poling process conducted during the in-situ XRD experiment, the macroscopic strain-electric field (S-E) loops were measured with a 2D digital image correlation (DIC) method under an optical microscope. A squareended bar specimen, derived from the same ceramic pellet used for the diffraction experiment, was placed in a custom-designed sample holder, where the lens of an optical microscope is focused on to the cross-section of the sample and the brass contact connected with the sample's electroded side in order to establish electrical contact to the HV amplifier. The specimen and contacts were immersed in Fluorinert ${ }^{\mathrm{TM}}$ FC-40 oil in order to avoid electrical arcing in air. Images were acquired using a Lumenera Infinity 2-1 M camera, controlled by a LabVIEW program, while a high voltage waveform was applied by means of a HP33120 function generator in combination with a HV amplifier (Chevin Research, Otley, UK). The values of applied voltage were recorded simultaneously with each image and subsequently used for plotting the strain-electric field (S-E) hysteresis loops. The sequence of images was processed with commercial DIC software (LaVision 
Davis 8.15 ) to determine the 2-D deformation relative to the initial zero-field reference image. Further details of the DIC experiment setup can be found in our previous study [23].

\section{Results and Analysis}

\subsection{Ferroelectric and dielectric properties}

In the present study, the BF-KBT-PT sample was poled by an electric field of 6 $\mathrm{kV} / \mathrm{mm}$ at a frequency of $2 \mathrm{~Hz}$, yielding a partially-saturated polarization-electric field ( $P$-E) loop, as shown in Figure 2 (a). The maximum polarization is $0.37 \mathrm{C} / \mathrm{m}^{2}$ and the coercive field is around $3.6 \mathrm{kV} / \mathrm{mm}$. In order to simulate the conditions of the in-situ XRD experiment, the macroscopic strain-electric field (S-E) loop was measured at $10 \mathrm{mHz}$, yielding a slightly lower coercive field of around $3.0 \mathrm{kV} / \mathrm{mm}$, compared with that the P-E loop, and a peak to peak longitudinal strain of about $3.3 \times 10^{-3}$, as shown in Figure 2 (b). The temperature-dependent dielectric permittivity was measured from room temperature up to $630^{\circ} \mathrm{C}$ at 1,10 and 100 $\mathrm{kHz}$. In the lower temperature range of $350-450{ }^{\circ} \mathrm{C}$, the dielectric maxima exhibit frequency-dependence, which is a characteristic dielectric behavior of relaxor ferroelectrics. At higher temperatures, another dielectric anomaly was observed at around $580{ }^{\circ} \mathrm{C}$. Further investigations are necessary to determine the structural origin of this phase transition.
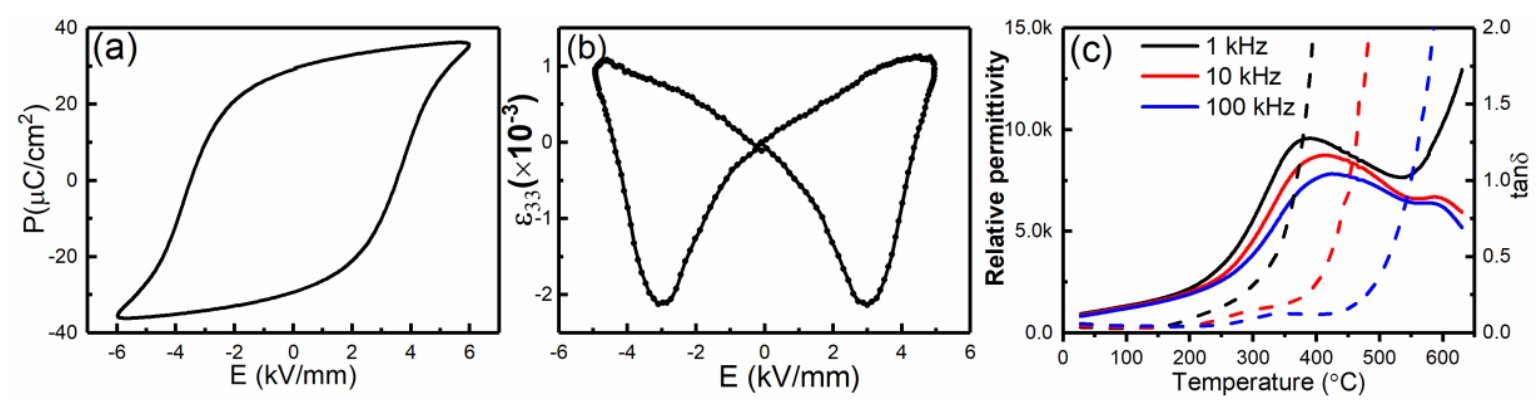

Figure 2. P-E, S-E loops and the temperature-dependent low-field dielectric properties of BF-KBT-PT ceramics in (a), (b) and (c) respectively. 


\subsection{In-situ high energy diffraction}

XRD contour plots of the $\{111\},\{200\}$ and $\{220\}$ reflections at $\psi=0^{\circ}$ are shown in Figure 3, including results recorded under two bipolar cycles of the electric field, from the initial unpoled state. Here, ' $\{\mathrm{hkl}\}$ ' is used to indicate the family of hkl reflections for the pseudo-cubic structure. Single (111), (002) and (022) peaks were initially observed for the unpoled state; on application of the electric field, the single (111) and (022) peaks split into doublets, while (002) remains singular, as shown in the line profiles of Figure 3 (d)-(f). These observations are consistent with previous reports from other NR systems [26] that exhibit an irreversible field-induced transformation into the long-range ordered ferroelectric state. In the present study, the initial crystal symmetry of cubic $P m \overline{3} m$ transformed to rhombohedral $R 3 c$, as confirmed by the full pattern refinement shown in Figure 6 below.

Evident variations in relative intensity with electric field were observed for the double-split peaks of $\{111\}$ and $\{220\}$ during the poling process, which can be attributed to the electric field-induced domain switching behavior (extrinsic effect), as reported previously in the NR state for NBT-based ceramics and rhombohedral PZT $[15,21]$. On the other hand, the single $\{002\}$ peak exhibits a shift with electric field, as shown in Figure $3(\mathrm{e})$, which indicates the development of tensile lattice strain (intrinsic effect) along $\langle 200\rangle$ for $\psi=0^{\circ}$. It has been demonstrated in previous work that the so-called 'intrinsic' lattice strain in $<200>$-oriented grains is also coupled to extrinsic domain switching strain in the surrounding polycrystalline matrix, due to elastic constraint and the associated inter-granular stresses $[11,19]$. There was also evidence of significant microstrain during and after the transformation, indicated by broadening of the $\{200\}$ diffraction peak. 

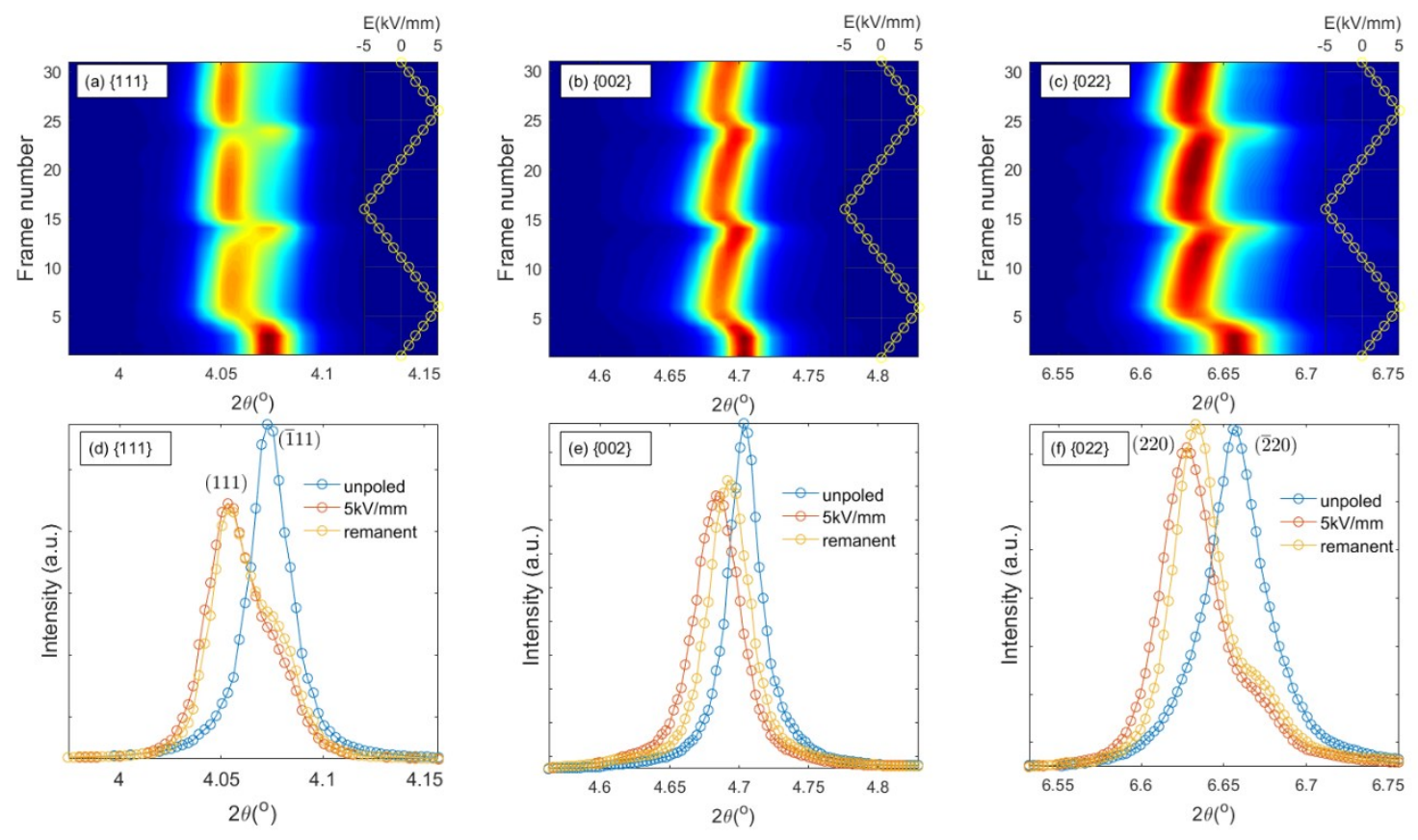

Figure 3. Contour plots of the $\{111\},\{200\}$ and $\{220\}$ reflections under 2 bipolar electric field cycles in (a), (b) and (c) respectively, and comparison of peak profiles for the unpoled state, under $5 \mathrm{kV} / \mathrm{mm}$ and in the remanent state in (d), (e) and (f).

Similar effects were also observed in the azimuthal angle-dependent XRD contour plots as shown in Figure 4. At $5 \mathrm{kV} / \mathrm{mm}$, there is clear evidence of peak splitting and intensity variations in the $\{111\}$ and $\{220\}$ reflections, whereas the $\{200\}$ reflection still remains singular and shifts continuously as $\psi$ changes from 0 to $90^{\circ}$. Thus, ferroelectric domain switching and lattice strain occur and exhibit an azimuthal angle-dependence, similar to the observations from previous reports of electric field-induced NR to FE phase transformations[15,21]. The intensities of the (111) and (220) reflections were enhanced at $0^{\circ}$ and suppressed at $90^{\circ}$ compared with their counterparts of the (111) and (220) reflections. Therefore, enhanced domain switching occurs along the direction closest to the electric field, accompanied by macroscopic elongation and contraction parallel and perpendicular to the electric field direction respectively, which also contributes to the remanent strains, $\varepsilon_{33}$ and $\varepsilon_{11}$, once the electric field is removed.

Therefore, the poling process leads to preferred domain orientation and anisotropic residual stress in the remanent state after the transformation from NR to FE. It is also noteworthy that the peak width of the $\{200\}$ reflection varies with 
the azimuthal angle due to the variations in the local inter-granular stresses; this could be evaluated further by micro-mechanical modelling[27]. The variations in domain orientation and lattice strain as a function of $\psi$ are quantified in terms of the domain and strain orientation distributions (DOD and SOD respectively), as detailed in section 3.4 below.
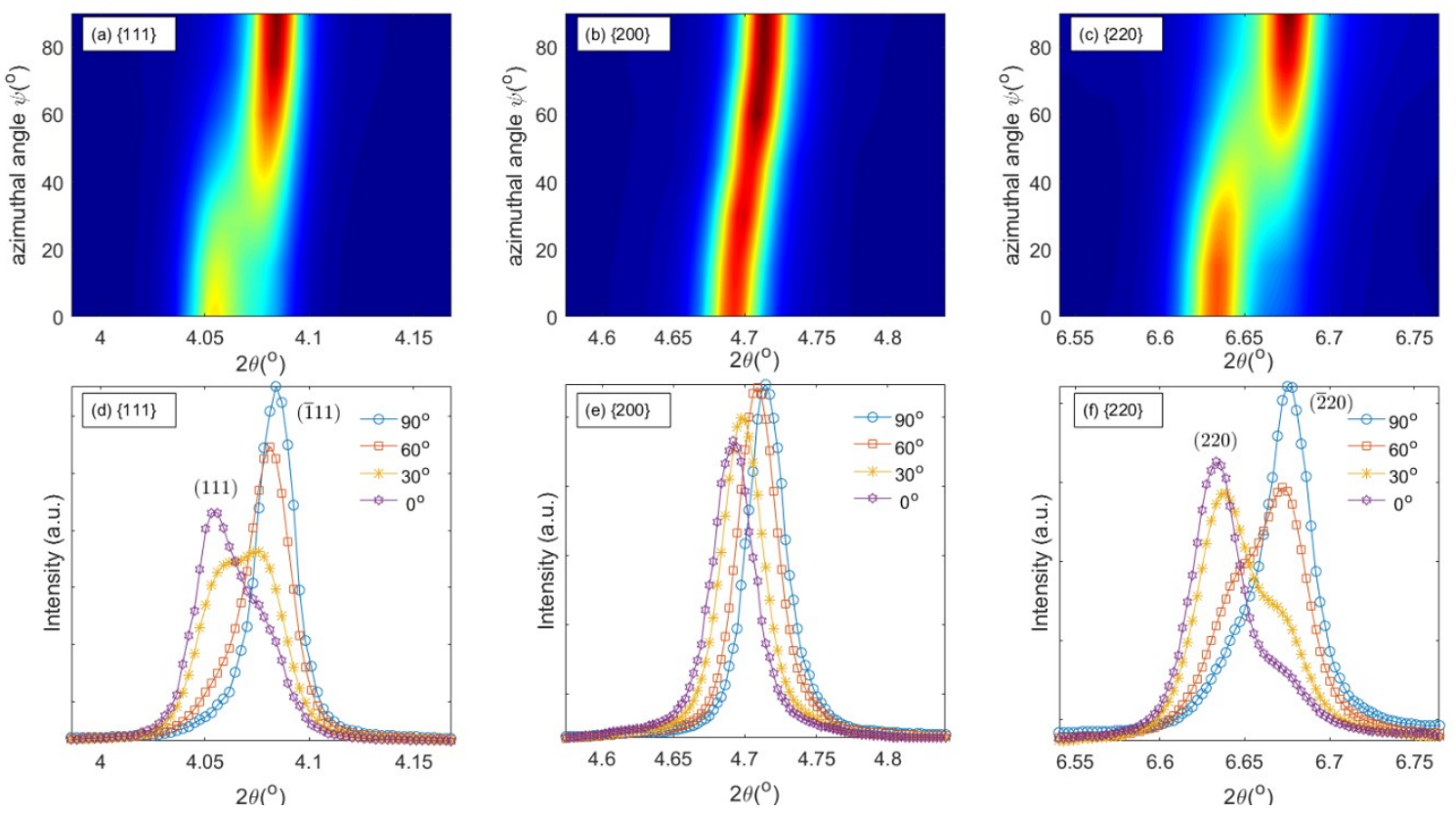

Figure 4. Contour plots of the $\{111\},\{200\}$ and $\{220\}$ XRD peak profiles against azimuthal angle $\psi$ measured at $5 \mathrm{kV} / \mathrm{mm}$ in (a), (b) and (c) respectively, and comparison of line profiles at $\psi=0^{\circ}, 30^{\circ}, 60^{\circ}$ and $90^{\circ}$ in (d), (e) and (f).

\subsection{XRD analysis by full-pattern fitting}

The observations of an electric field-induced phase transformation, strain anisotropy and texture introduce difficulties for the implementation of full pattern refinement without suitable models to account for the texture and anisotropic lattice strain. Full-pattern fitting was applied initially to illustrate the importance of models incorporating texture and anisotropy effects. As shown in Figure 5 (a), the XRD pattern of the unpoled sample can be fitted directly with the cubic structure, $P m \overline{3} m$, using full-pattern refinement. A slight misfit is apparent in the intensity for $\{111\}$, which is attributed to anisotropic peak broadening. The XRD pattern for the poled sample was refined as rhombohedral phase, $R 3 C$, as shown in Figure 5 
(b), using $\psi=54.45^{\circ}$ as the invariant angle (indicating the strain-free state) determined according to the method described section 3.4 below.

The XRD patterns for the poled sample at $\psi=0^{\circ}$ and $90^{\circ}$ were also refined as rhombohedral phase, $R 3 c$, as shown in Figure 5 (c) and (d), using the fixed lattice parameters of the strain-free state and a second-order spherical harmonic function to describe crystallographic texture [28], but without considering the anisotropic strain and size effects. In both cases, clear differences are observed between the experimental and calculated results, particularly for $\psi=0^{\circ}$, as shown in Figure 5 (c). These results demonstrate the errors that may be introduced if the obvious anisotropic lattice strain effects are neglected. In particular, the evident shifts in peak position for the (111) and (220) reflections at $\psi=0^{\circ}$, compared with those at $\psi=54.45^{\circ}$ and $90^{\circ}$, lead to a strong misfit between the experimental data and calculated profiles, which cannot be corrected using solely a preferred orientation function.



Figure 5. XRD patterns for (a) unpoled and (b)-(d) poled BF-KBT-PT samples for (b) $\psi=54.45^{\circ}$, (c) $\psi=0^{\circ}$ and (d) $\psi=90^{\circ}$ with full-pattern refinement incorporating a second order spherical harmonic function for texture at $\psi=0$ and $90^{\circ}$. Vertical dashed lines indicate positions of diffraction peaks fixed according to the refinement of the pattern at the strain-free invariant angle, $\psi=54.45^{\circ}$. 
For comparison, a full pattern refinement was also conducted using MAUD [25], adopting the WSODF Popa-Balzar model and a second order Harmonic function to account for anisotropic strain and texture respectively $[29,30]$. Due to the uniaxial poling process, a fibre texture was adopted for the refinement[31]. The fitted results of full pattern refinement for the remanent state at selected azimuthal angles, $\psi=0^{\circ}, 30^{\circ}, 60^{\circ}$ and $90^{\circ}$, are shown in Figure 6 . Although the fitted results are well-matched with the experimental data, the refinement process became more tedious and time-consuming after introducing the physical models in combination with the texture and anisotropy functions. Therefore, in principle single profile fitting could provide a more efficient method to extract the domain orientation distribution (DOD) and strain orientation distribution (SOD) functions from a limited number of representative reflections such as $\{111\},\{200\}$ and $\{220\}$. However, it should be noted that the full pattern refinement is still the most appropriate method for solving the multiple phases and overlapping peak problems.

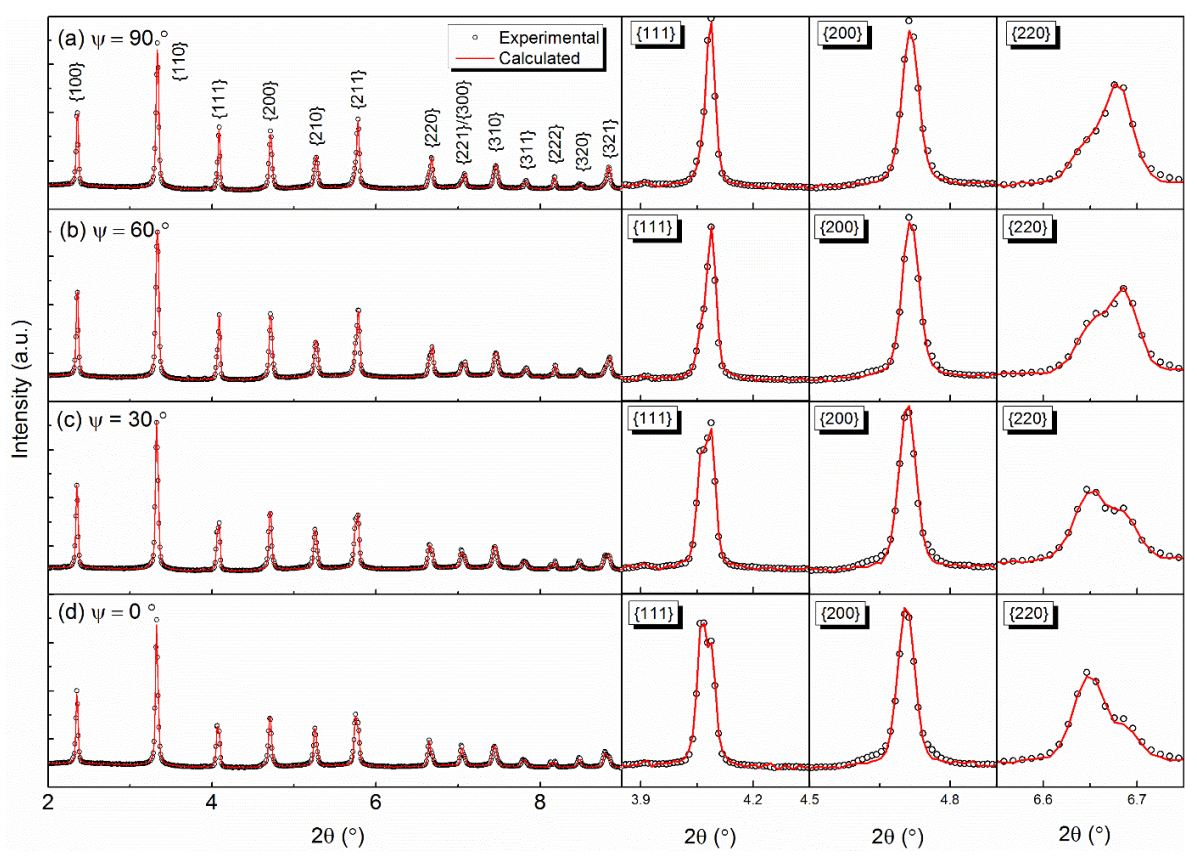

Figure 6. Experimental and calculated XRD patterns of BF-KBT-PT ceramics in the remanent state for azimuthal angles of $90,60,30$ and $0^{\circ}$ in (a), (b), (c) and (d) respectively; the peak profiles of $\{111\},\{200\}$ and $\{220\}$ reflections are also presented. Full pattern profiles were calculated using MAUD, incorporating models for both the SOD and DOD functions [26]. 
The SOD for the $<002>$ pole and DOD for the (111) and (220) reflections were extracted from the full-pattern refinement. The DODs are expressed in terms of the multiple of a random distribution (MRD). Both the SOD and DODs exhibit azimuthal angle-dependence, with the highest and lowest levels of strain and MRD values being observed at $\psi=0^{\circ}$ and $90^{\circ}$ respectively, where the multiple of random distribution (MRD) is a measure of the degree of preferred domain orientation relative to that of a random distribution and is defined by Eqs. 17 and 18 below. The results obtained for the DODs, retrieved from the full pattern refinement with MAUD, are subsequently compared with the results from single profile fitting in the following section, as shown in Figure 11 below.

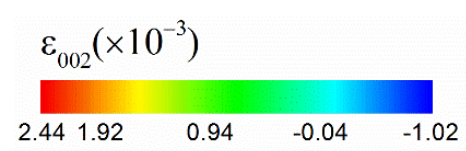

(a)

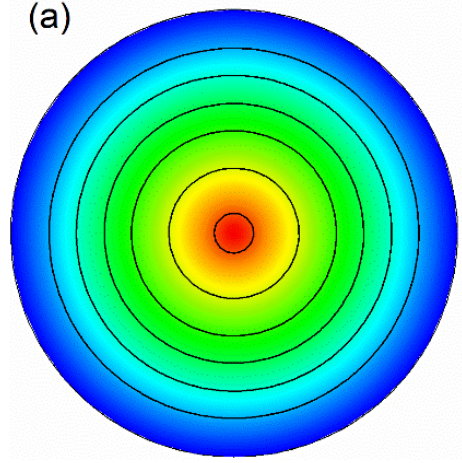





(c) (220)

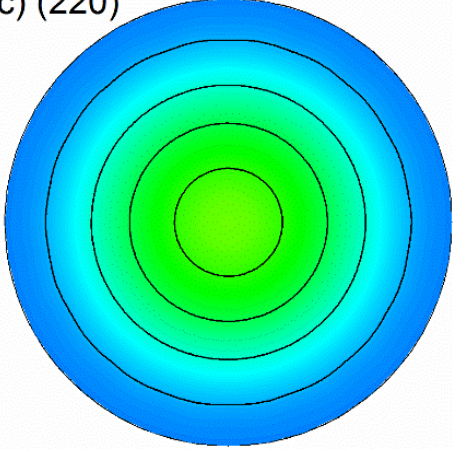

Figure 7. The (a) strain orientation distribution (SOD) for the (002) pole and domain orientation distributions (DODs) for (b) (111) and (c) (220) poles respectively, expressed in terms of the Multiple of Random Distribution (MRD), retrieved from full pattern refinement of the poled sample in the remanent state.

\subsection{Methodology for combined single profile fitting}

In this section, the methods developed for the evaluation of crystallographic information (d-spacings and electric field-induced texture) according to the measured XRD peak profiles (peak position and intensities) are discussed in detail. Before proceeding with the discussion, it should be noted that the rhombohedral inter-axial angle, $\alpha$, and the spontaneous strain, $\varepsilon_{s}=\frac{d_{111}-d_{\overline{111}}}{d_{\overline{111}}}$, of the BF-KBT-PT ceramic are around $89.74^{\circ}$ and $6.2 \times 10^{-3}$, as listed in Table 1 . The assumption that 
we express the elastic strain along [002] as the change in the (002) lattice spacing $d_{(002)}$ is not exactly true for the rhombohedral structure. However, we can neglect the fact that the [002] direction (for example) is not precisely normal to the (002) plane, due to the very small discrepancy between $89.74^{\circ}$ and $90^{\circ}$. In other words, the error introduced in the measurement of the $<002>$ strain by this assumption is extremely small.

Assuming the BF-KBT-PT ceramic to be elastically isotropic and applying the Eshelby theory, the elastic poling strain $\varepsilon_{(002)}^{P}$ along $<002>$, caused by ferroelectric domain switching in the surrounding polycrystalline matrix is given by[32]:

$$
\varepsilon_{(002)}^{P}(\psi)=\frac{(7-5 v)}{15(1-v)}\langle\varepsilon\rangle\left(\frac{3}{2} \cos ^{2} \psi-\frac{1}{2}\right)
$$

where $\langle\varepsilon\rangle$ is the elongation along the poling direction and $v$ is Poisson's ratio. The volume fraction of [111]-oriented domains, $\eta_{111}$, can also be expressed according to a second order spherical harmonic function proposed by Hall et al.[19]:

$$
\eta_{111}(\psi)=\frac{1}{4}+\left(\eta_{111}\left(0^{\circ}\right)-\frac{1}{4}\right)\left(\frac{3}{2} \cos ^{2} \psi-\frac{1}{2}\right)
$$

In other words, concerning the FE rhombohedral phase, both the $\varepsilon_{(002)}^{P}(\psi)$ and $\eta_{111}(\psi)$ functions exhibit the form $a+b \cos ^{2} \psi$. However, the information extracted directly from the XRD peak profile fitting comprises the d-spacing and integrated intensity of each reflection, which needs further processing to determine the desired lattice strain and domain fractions. For example, the lattice strain $\varepsilon_{002}$ is given by:

$$
\varepsilon_{002}=\frac{d_{002}-d_{002}^{0}}{d_{002}^{0}}
$$

where $d_{200}^{0}$ is the $d$-spacing in the unpoled state. According to the linear relationship between $d_{002}$ and $\varepsilon_{002}, d_{002}(\psi)$ can also be fitted with a function of the form $a+b \cos ^{2} \psi$. Therefore, the invariant angle, $\psi_{0}$, and the lattice spacing, $d_{200}^{0}$, of the unpoled state for the electric field-induced rhombohedral polycrystal can be determined by fitting $d_{002}(\psi)$ across the azimuthal angle range under different electric field levels. Afterwards, the $\varepsilon_{002}$ values can be evaluated 
according to Eq. 10.

Compared with the evaluation process for $\varepsilon_{002}$, the quantification of electricfield induced texture is more complex. The preferred orientation of ferroelectric domains is quantified in terms of the domain fractions, given by Eq. 11 and 12 for the (111) and (220) poles of the FE rhombohedral phase respectively:

$$
\begin{aligned}
& \eta_{111}=\frac{I_{111}}{I_{111}+3 \frac{I_{111}^{0}}{I_{111}^{0}} \times I_{111}} \\
& \eta_{220}=\frac{I_{220}}{I_{220}+\frac{I_{220}^{0}}{I_{220}^{0}} \times I_{220}}
\end{aligned}
$$

where $I_{h k l}$ is the measured intensity from the XRD peak profile and the superscript ' 0 ' indicates the reference unpoled state[33]. The introduction of the reference intensity ratios $\gamma_{111}^{0}=\frac{I_{111}^{0}}{I_{111}^{0}}$ and $\gamma_{220}^{0}=\frac{I_{220}^{0}}{I_{220}^{0}}$ in Eq. 11 and 12, corresponding to the unpoled (randomly-oriented) state, were first proposed by Jones et al. in order to compensate for differences in structure factors between (111)/ (111) and $(220) /(2 \overline{2} 0)$ pairs of reflections [33]. However, the values of $\gamma_{111}$ and $\gamma_{220}$ cannot be determined directly from the textured electric field-induced rhombohedral phase at $\psi=0^{\circ}$ for example. Instead, the information derived directly from peak fitting is the ratio $R_{h k l}$, which describes the relative intensities of the corresponding reflections, given in Eq. 13 and 14 for the reflections (111) and (220) respectively:

$$
\begin{aligned}
& R_{111}=\frac{I_{111}}{I_{111}+I_{\mathrm{I} 11}} \\
& R_{220}=\frac{I_{220}}{I_{220}+I_{220}}
\end{aligned}
$$

It is evident that the intensity ratio, $R_{h k l}$, and domain fraction, $\eta_{h k l}$, are related; for example, in the case of the $\{111\}$ family, according to Eq. 11 and $13, \eta_{111}$ and $R_{111}$ are related by:

$$
\eta_{111}=\frac{1}{1+3\left(\frac{1}{R_{111}}-1\right)}
$$


As noted above, the domain fraction, $\eta_{111}$, satisfies a second-order spherical harmonic function of the form $a+b \cos ^{2} \psi$ according to Eq. 9, while the intensity ratio $R_{111}$ follows a nonlinear relationship with $\cos ^{2} \psi$ given by:

$$
R_{111} \propto \frac{1}{\frac{1}{a+b \cos ^{2} \psi}+c}
$$

Therefore, the reference intensity ratio, $\gamma_{111}^{0}$, can be solved by introducing the invariant angle, $\psi_{0}$, into Eq. 16 , where the corresponding fitting parameters $(a, b$ and c) can be determined by the nonlinear fitting of experimental data for $R_{111}$. Afterwards, $\gamma_{111}^{0}$ can be employed for the calculation of $\eta_{111}$.

The evaluation process used to determine the crystallographic parameters of the electric field-induced rhombohedral phase in the unpoled state is shown by the flow chart presented in Figure 8. The data analysis process starts from fitting of single $\{200\}$ or double $\{111\}$ and $\{220\}$ peak profiles using a pseudo-Voigt function in MATLAB. The results obtained for peak positions and integrated intensities are then transformed into the corresponding lattice spacing ( $d$-spacing) and domain orientation fractions. The strain free d-spacing, $d_{h k l}^{0}$, and invariant angle, $\psi_{0}$, can be identified from the intersection of the $d_{002}(\psi)$ fitted profiles under different electric field levels. Afterwards, the invariant angle can be introduced into the nonlinear R- $\psi$ relationships, fitted according to Eq. 16, in order to solve the intensity ratios for the unpoled state. Due to limited variations in the lattice spacings of the double-split peaks, the $d_{0}$ values associated with these peaks at the invariant angle were estimated by linear interpolation of the corresponding d-spacings at azimuthal angles of $45^{\circ}$ and $60^{\circ}$.

The reference intensity ratios for the unpoled state were applied to the calculation of the domain fractions, and then the electric field-induced macroscopic strain was estimated based on the resulting d-spacing and domain fractions. In the present study, the macroscopic strain was evaluated by two different methods, which are firstly compared with each other and then with the S-E loop measured by DIC. Firstly, Daymond's method was employed to estimate the macroscopic strain, in addition to quantifying the effective lattice strain and evaluating the 
strain anisotropy between different oriented grain families [34]. The second method, proposed by Jones et al.[13], involves analysis of the polar $<111>$ domain orientation distribution (DOD) function, together with the lattice strain, and has the advantage that it is possible to distinguish and quantify the contributions of intrinsic and extrinsic mechanisms to macroscopic strain. The peak profile analysis process was conducted using a MATLAB script developed by the authors, while further full pattern refinement incorporating models for the DOD and SOD functions was conducted using MAUD[35] in order to check the validity and compare with the methods developed in the present study.



Figure 8 Flow chart of data analysis procedures including: determination of the location of invariant angle, $d$-spacings and MRD of strain free state for $\{111\}$, $\{200\}$, and $\{220\}$, and the calculation of macroscopic strain based on different methods, in order to perform analysis of anisotropy and evaluate intrinsic and extrinsic contributions.

\subsection{Determination of the invariant angle}

The $\mathrm{d}_{002}-\psi$ and $\varepsilon_{002}-\psi$ data obtained over a range of electric field values are 
presented in Figure 9(a) and (b) respectively. Values calculated by nonlinear fitting according to a squared cosine function are also included as the solid lines in these figures. The fitted data obtained under different electric field levels intersect at almost the same positions, with average azimuthal angles for the intersection points around $54.45^{\circ}, 125.55^{\circ}, 234.55^{\circ}$ and $305.55^{\circ}$. Therefore, the aforementioned invariant angle, $\psi_{0}$, was determined as $54.45^{\circ}$, and the d-spacing of the unpoled state, $d_{002}^{0}$, was estimated as $1.986 \AA$. The invariant angle derived from this procedure is in good agreement with the value of $54.7^{\circ}$, predicted on the basis of a volume-conserving domain switching mechanism and elastic isotropy [12]. It is notable that the invariant angle does not vary significantly at electric field levels up to $5 \mathrm{kV} / \mathrm{mm}$, indicating that the piezoelectric behavior of the BF-KBT-PT ceramics is also relatively isotropic, i.e. $d_{31} \approx-\frac{1}{2} d_{33}$.

Table 1 The lattice parameters of both cubic and rhombohedral phases, and the intensity ratios for $\{111\}$ and $\{220\}$ reflections of rhombohedral phase, obtained by Rieveld refinement with Topas using the XRD profiles of the unpoled state at $\psi=0^{\circ}$ and the poled state at $\psi=54.45^{\circ}$ respectively.

\begin{tabular}{|c|c|c|c|c|c|c|}
\hline Phase & $\mathbf{a}(\boldsymbol{\AA})$ & $\boldsymbol{\alpha}\left(^{\circ}\right)$ & Volume $\left(\AA^{\mathbf{3}}\right)$ & $\boldsymbol{I}_{\mathbf{1 1 1}}^{\mathbf{0}} / \boldsymbol{I}_{\mathbf{1 1 1}}^{\mathbf{0}}$ & $\boldsymbol{I}_{\mathbf{2 2 0}}^{\mathbf{0}} / \mathbf{I}_{\mathbf{2 2 0}}^{\mathbf{0}}$ & $\boldsymbol{\varepsilon}_{\boldsymbol{s}}$ \\
\hline Cubic & 3.968 & 90 & 62.476 & & & \\
\hline Rhombohedral & 3.976 & 89.74 & 62.419 & 0.263 & 0.673 & $6.2 \times 10^{-3}$ \\
\hline
\end{tabular}

The changes in strain orientation distribution (SOD) as a result of the applied electric field were subsequently calculated according to Eq. 10 and are presented as a contour map in Figure 9 (c). It is evident that $\varepsilon_{002}$ varies in response to the electric field, exhibiting hysteretic behavior. The SOD also exhibits a gradual variation with azimuthal angle from a positive longitudinal strain $\sim 4.0 \times 10^{-3}$ at $\psi=0^{\circ}$ to a negative transverse strain $\sim-2.0 \times 10^{-3}$ at $\psi=90^{\circ}$, depending on the magnitude of the electric field. Furthermore, $\varepsilon_{002}$ remains invariant around $\psi_{0}$ in agreement with the argument presented above. The identified invariant angle, $\psi_{0}$, is also relatively independent of the electric field level and stress state, which is consistent with the previously reported physical laws and diffraction 

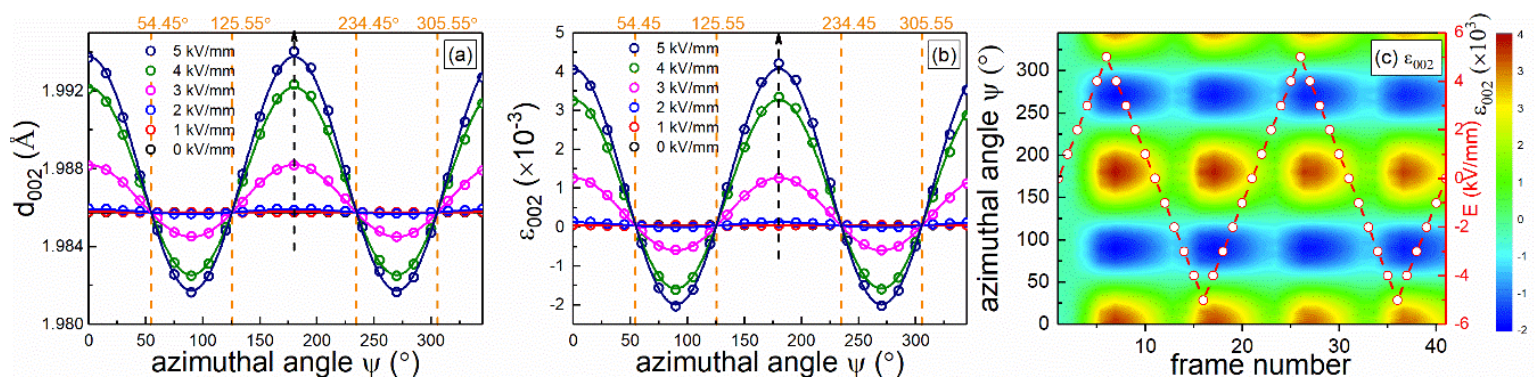

Figure 9. (a) $d_{002}-\psi$ and (b) $\varepsilon_{002}-\psi$ relationships under an increasing electric field from the initial unpoled state to $5 \mathrm{kV} / \mathrm{mm}$. (c) Contour plot of lattice strain, $\varepsilon_{002}$, for $\psi=0$ to $345^{\circ}$, during the electric field cycling procedure.

\subsection{Texture analysis}

The experimental data for the peak intensity fractions $R_{111}$ and $R_{220}$ were fitted to a nonlinear function according to Eq. 16, as shown in Figure 10 (a) and (d) respectively. The reference intensity ratios, $\gamma_{111}^{0}$ and $\gamma_{220}^{0}$, were determined from the remanent state data in order to avoid potential volume variation or intergranular stress at high electric field levels. The fitted line profiles of $R_{111}$ and $R_{220}$ are well matched with the experimental data. The reference state intensity ratios, $\gamma_{111}^{0}$ and $\gamma_{220}^{0}$, were determined as 0.268 and 0.692 respectively. Furthermore, the field-dependent $\eta_{111}$ and $\eta_{220}$ values were then calculated according to Eq. 11 and 12, providing a measurement of the DODs throughout the whole cycling procedure.

In order to provide a common measurement of the invariant angle location, the domain fractions were transformed into the corresponding MRD (multiple of random distribution) values, where the MRD is equal to unity for both (111) and (220) poles in the reference randomly-oriented state. The relationships between the $M R D_{h k l}$ and $\eta_{h k l}$ values are:

$$
\begin{aligned}
& M R D_{111}=4 \eta_{111} \\
& M R D_{220}=2 \eta_{220}
\end{aligned}
$$


The DODs, expressed as $M R D_{111}$ and $M R D_{220}$, for electric field levels from 5 to -2 $\mathrm{kV} / \mathrm{mm}$, are presented in Figure $10(\mathrm{~b})$ and (e) together with the corresponding calculated functions, fitted according to a squared cosine relationship. The experimental data are well matched with the fitted profiles, and intersect each other around $54.45^{\circ}$, with corresponding MRD values close to 1 as indicated by the red dashed line.

Furthermore, the DODs also vary periodically in response to the electric field, as shown in the contour plots in Figure 10(c) and (f). At $\psi=0^{\circ}$, the domain texture appeared abruptly after the transformation into the rhombohedral phase. The MRD value reaches a maximum at $5 \mathrm{kV} / \mathrm{mm}$ and then decreases slowly until reaching the coercive field in another half cycle, whereas the opposite trend is observed for $\psi=90^{\circ}$. The domain switching effects were gradually enhanced within the first cycle according to the observation of $M R D_{111}$ at $\psi=0^{\circ}$ and $180^{\circ}$, and stabilised within the second cycle. After poling, the domains are aligned with their [111] polar axes along the direction closest to that of the electric field. It is also noteworthy that the MRD values are almost invariant and close to 1 for both (111) and (220) reflections, forming a clear boundary in the contour plots, at the previously derived invariant angle around $54.45^{\circ}$ (and the equivalent points of $125.55,234.45$ and $305.55^{\circ}$ ). 

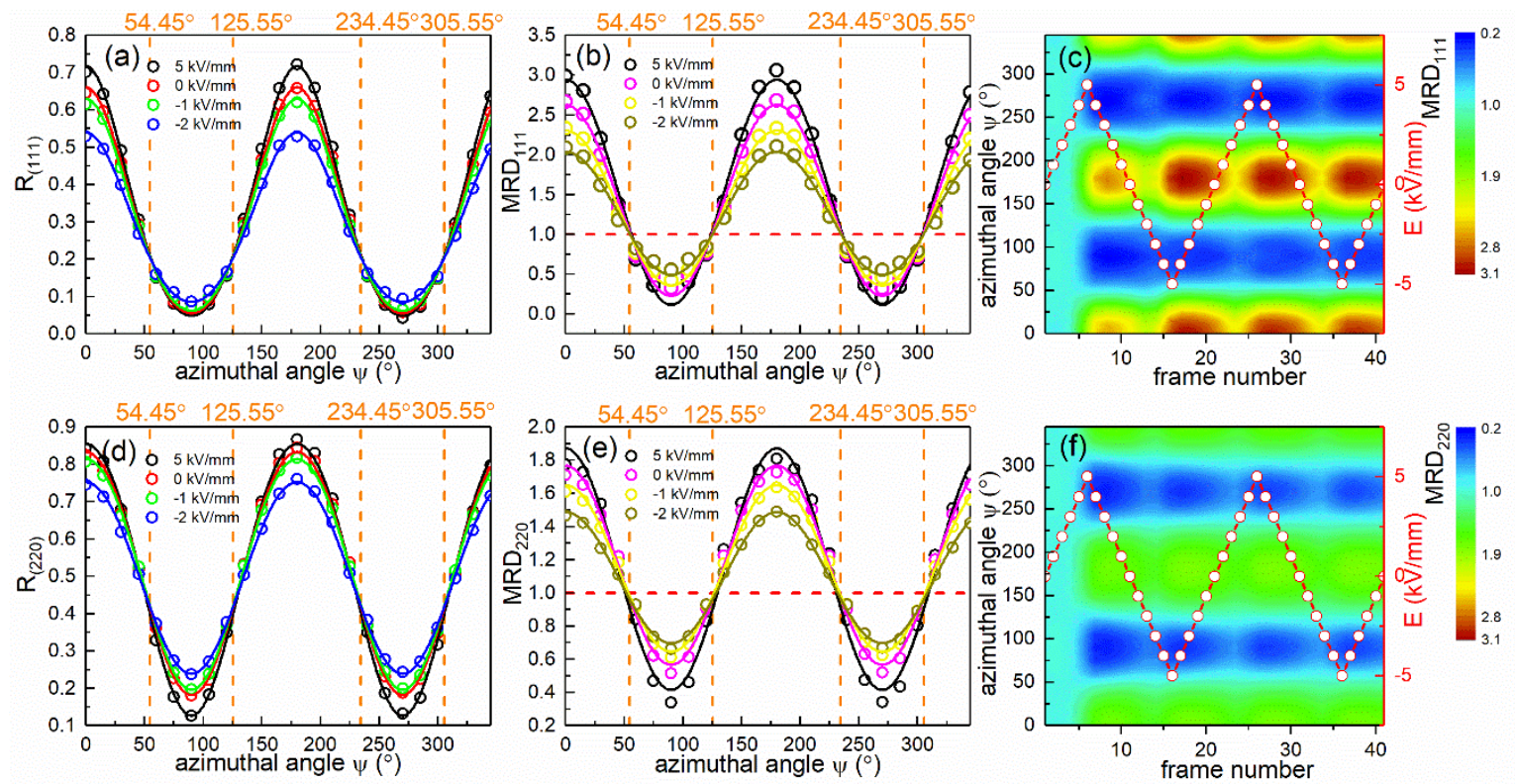

Figure 10. Variation in intensity fractions (a) $R_{111}$ and (d) $R_{220}$ as a function of $\psi$ at electric field levels from 5 to $-2 \mathrm{kV} / \mathrm{mm}$. The derived $M R D_{111}$ and $M R D_{220}$ values obtained at electric field levels from 5 to $-2 \mathrm{kV} / \mathrm{mm}$ are shown in (b) and (e) respectively, where the red dashed lines indicate the MRD value of unity. Contour plots for $M R D_{111}$ and $M R D_{220}$ covering the whole electric cycling procedure are shown in (c) and (f) respectively.

\section{Discussion}

\subsection{Comparison of results from full-pattern and single profile fitting procedures}

In order to examine the validity of the method described in section 3.4 above, the resulting DODs were compared with those derived from the full-pattern fitting procedure using MAUD, yielding the results shown in Figure 11 (a). The azimuthal angle-dependence of $M R D_{111}$ and $M R D_{220}$ obtained from peak profile fitting are in good agreement with the results from full pattern refinement. The results reinforce the need for a realistic physical model for strain anisotropy and texture in full pattern refinement, which can also be solved by individual peak profile fitting. It is also noteworthy that, for both methods, the intersection points of linear fits 
for $M R D_{111}$ and $M R D_{220}$ against $\cos ^{2} \psi$ occur around $\cos ^{2} \psi=\frac{1}{3}\left(\psi_{0} \approx 54.7^{\circ}\right)$ with MRD values equal to 1 , which also confirms the presence of a consistent invariant angle for differently-oriented grain families. Furthermore, the maximum of $M R D_{111}$ is approximately twice that of $M R D_{220}$. It is also evident that the intrinsic (lattice strain) contribution is significantly larger for $\varepsilon_{\{220\}}$ than for $\varepsilon_{\{111\}}$, as illustrated in Figure 11 (b) and (c). This point is discussed further in section 4.3 below combined with the strain anisotropy analysis.
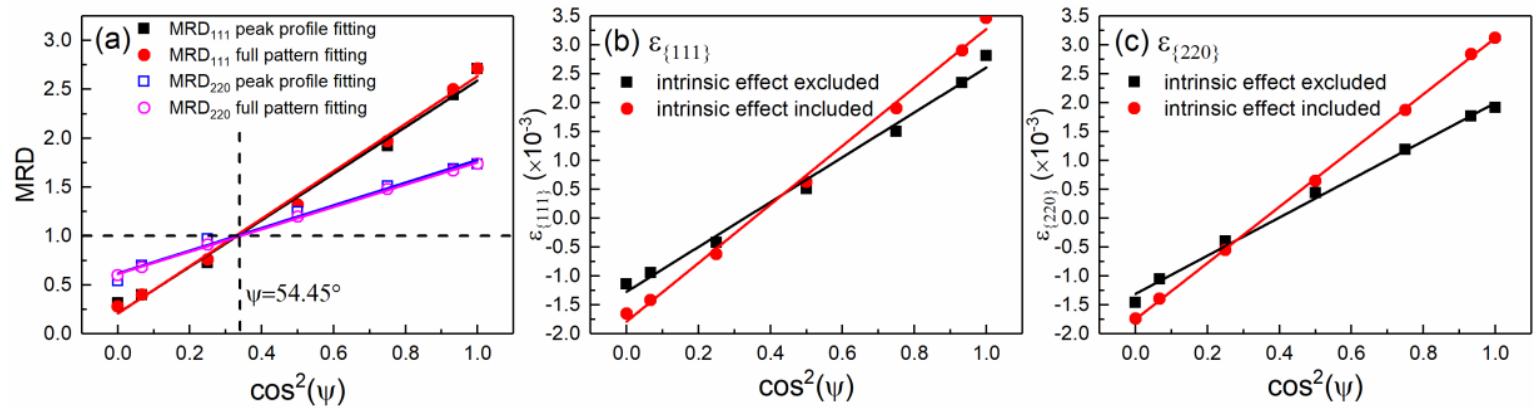

Figure 11. (a) Comparison of the remanent DODs obtained by peak profile fitting and full pattern fitting, expressed in terms of $M R D_{111}$ and $M R D_{220}$. (b) and (c) Effective lattice SODs of $\varepsilon_{\{111\}}$ and $\varepsilon_{\{220\}}$, both with and without the intrinsic (lattice strain) contributions.

\subsection{Estimation of macroscopic strain}

The macroscopic strain induced by the applied electric field can be effectively quantified according to Eq. 6, including contributions from the phase transformation, intrinsic lattice strain and extrinsic domain switching. The volumes of the pseudocubic and rhombohedral unit cells are very close to each other, determined as 62.476 and $62.419 \AA^{3}$ respectively (Table 1 ). The resulting volumetric and linear transformation strains, $\varepsilon_{V}^{\text {trans }}$ and $\varepsilon_{33}^{\text {trans }}$, are only about $0.9 \times 10^{-3}$ and $-0.3 \times 10^{-3}$ respectively. The linear transformation strain, $\varepsilon_{33}^{\text {trans }}$, is one order of magnitude smaller than the macroscopic axial strain, $\varepsilon_{33}$. Therefore, the poling process and phase transformation in the present study can be assumed as approximately volume conserving. As described above, the macroscopic strain will be evaluated by two different methods, which emphasize different aspects of the analysis. The first method is denoted as the Daymond method (or the weighted 
average method), which transforms the lower-order symmetry to an effective cubic symmetry and benefits from the analysis of anisotropic strain and elastic coupling of differently-oriented grain families[34]. The second method, devoted as the Jones method, has the ability to distinguish between the contributions from both the intrinsic (lattice strain) and extrinsic (domain switching) mechanisms to the macroscopic strain. The principles of these two methods are outlined in our previous report[24].

Initially, the Daymond method was adopted, taking account of the lattice strains for the representative grain orientations $\{111\},\{200\}$ and $\{220\}$. The domain switching contribution was incorporated into an effective lattice strain, $d_{\{h k l\}}^{*}$, through a weighted-averaging process that incorporates both the lattice spacing and corresponding domain fractions according to the following equations for $d_{\{111\}}^{*}$ and $d_{\{220\}}^{*}$ respectively:

$$
\begin{aligned}
& d_{\{111\}}^{*}=\eta_{111} \times d_{111}+\left(1-\eta_{111}\right) \times d_{\overline{1} 11} \\
& d_{\{220\}}^{*}=\eta_{220} \times d_{220}+\left(1-\eta_{220}\right) \times d_{022}
\end{aligned}
$$

The strain for each grain family was then calculated according to the following relation:

$$
\varepsilon_{\{h k l\}}=\frac{d_{\{h k l\}}-d_{\{h k l\}}^{0}}{d_{\{h k l\}}^{0}}
$$

Then the average macroscopic strain for $\psi=0^{\circ}$, for example, was determined by taking into account the multiplicity $m_{\{h k l\}}$ of the corresponding $\langle h k l\rangle$-oriented grains:

$$
\varepsilon_{33}^{t o t a l}=\frac{\sum m_{\{h k l\}} \varepsilon_{\{h k l\}}}{\sum m_{\{h k l\}}}
$$

In the present study, $\varepsilon_{33}^{\text {total }}$ was estimated as:

$$
\varepsilon_{33}^{\text {total }}=\frac{m_{\{111\}} \varepsilon_{\{111\}}+m_{\{200\}} \varepsilon_{\{200\}}+m_{\{220\}} \varepsilon_{\{220\}}}{m_{\{111\}}+m_{\{200\}}+m_{\{220\}}}
$$

where the multiplicities are 8,6 and 12 for $m_{\{111\}}, m_{\{200\}}$ and $m_{\{220\}}$ respectively. 
In the weighted-averaging process, the lattice strain and domain switching contributions cannot be effectively distinguished, since they are combined in the averaging process. However, the contributions to the macroscopic strain from each grain orientation are quantified, providing key information for the analysis of strain anisotropy. Although the diffraction data provide strain and texture information over the full range of grain orientations, the results reported here for the Daymond method focus on the strain data for $\psi=0^{\circ}$ and $90^{\circ}$, yielding the macroscopic strain components $\varepsilon_{33}^{\text {total }}$ and $\varepsilon_{11}^{\text {total }}$ respectively.

The electric field-induced macroscopic strain can also be separated into intrinsic (lattice strain) and extrinsic (domain switching) contributions using the Jones method[13]. The respective contributions of intrinsic strain, $\varepsilon_{33}^{\text {int }}$, and extrinsic strain, $\varepsilon_{33}^{e x t}$, are evaluated according to Eqs. 24 and Eq. 25 respectively. The intrinsic lattice strains, are weighted by the lattice strain associated with each crystal plane, $\varepsilon_{\{h k l\}}$, at $\psi=0^{\circ}[34]$ according to:

$$
\varepsilon_{33}^{i n t}=\frac{\sum_{h k l} \eta_{h k l}(\psi=0) m_{h k l} \varepsilon_{h k l}(\psi=0)}{\sum_{h k l} \eta_{h k l}(\psi=0) m_{h k l} \varepsilon_{h k l}}
$$

The extrinsic effect associated with domain switching is effectively quantified by the accumulated contribution of the $\{111\}$ domain orientation for azimuthal angles from $0^{\circ}$ to $90^{\circ}$ according to the sample symmetry proposed by Jones [13]. Therefore, the extrinsic strain is obtained by integrating the projection of the spontaneous strain within the orientation space, as given by following equation:

$$
\varepsilon_{33}^{e x t}=\varepsilon_{s} \int_{0}^{\frac{\pi}{2}}\left[m \Delta \eta(\psi) \cos ^{2}(\psi)\right] \sin (\psi) d \psi
$$

where the spontaneous strain of the rhombohedral phase, $\varepsilon_{s}$, and the product of the multiplicity $m$ and domain switching fraction, $\Delta \eta$, for the (111) reflection, are given as:

$$
\begin{gathered}
\varepsilon_{S}=\frac{d_{111}-d_{\overline{1} 11}}{d_{\overline{11} 1}} \\
\Delta \eta=\frac{\left(M R D_{111}-1\right)}{4}
\end{gathered}
$$

Hence, the intrinsic and extrinsic strains can be evaluated to help understand their 
contributions to the macroscopic strain, $\varepsilon_{33}^{\text {total }}$.

The calculated $\varepsilon_{33}^{\text {total }}$ values based on these two different methods exhibit consistent agreement, as shown in Figure 12 (a). Excluding the pseudocubic region before the phase transformation occurred, the calculated $\varepsilon_{33}^{\text {total }}$ values for both methods exhibit similar values and the same trends. However, a slight difference in the magnitudes of the calculated $\varepsilon_{33}^{\text {total }}$ values originates from the different approaches, where the weighted-average method mainly focuses on the effective lattice strain and may underestimate domain switching effects by the adoption of only three different grain families (two of them exhibiting noncentrosymmetric features), in order to represent all the differently-oriented grains.

The deconvoluted intrinsic, $\varepsilon_{33}^{\text {int }}$, and extrinsic, $\varepsilon_{33}^{\text {ext }}$, contributions to $\varepsilon_{33}$ behave quite differently with respect to the applied electric field, as shown in Figure 12 (b). It is noteworthy that $\varepsilon_{33}^{\text {int }}$ (around $2.75 \times 10^{-3}$ at $5 \mathrm{kV} / \mathrm{mm}$ ) dominates during the first half cycle before $\varepsilon_{33}^{e x t}$ reaches a stable state during the following poling process. Therefore, the phase transformation is a dynamic and competitive process for both intrinsic and extrinsic contributions before reaching a stable state, where the extrinsic effect is gradually enhanced during the two complete cycles of the electric field. However, the intrinsic strain is still dominant with a major contribution after the first half cycle (around $2.5 \times 10^{-3}$ at $5 \mathrm{kV} / \mathrm{mm}$ ) compared with the extrinsic strain (around $1.5 \times 10^{-3}$ at $5 \mathrm{kV} / \mathrm{mm}$ ); this is somewhat different to previous observations in normal ferroelectrics, where the extrinsic contributions tend to be more pronounced or close to that of the intrinsic effect[17,21]. Furthermore, $\varepsilon_{33}^{\text {int }}$ varies more sensitively with the applied electric field compared with the corresponding behavior of $\varepsilon_{33}^{\text {ext }}$, which exhibits a tendency for saturation. Upon field reduction, the magnitude of $\varepsilon_{33}^{\text {int }}$ decreases significantly with the gradual removal of electric field and reaches a similar level to $\varepsilon_{33}^{\text {ext }}$ around $1.4 \times 10^{-3}$ in the remanent state. Variations in the extrinsic strain, triggered around each excursion past the coercive field, are evidence of domain switching and contribute to the nonlinearity and hysteresis of the macroscopic strain behaviour. It is also noteworthy that the intrinsic contribution dominate the electric-field induced macroscopic strain in accordance with the rhombohedral spontaneous 
strain $6.2 \times 10^{-3}$, which is consistent with the observed intrinsic and extrinsic effect constitutions (61\% and 39\%) of rhombohedral PZT 54/46 (spontaneous strain $6.8 \times 10^{-3}$ ) close to the MPB region[37].
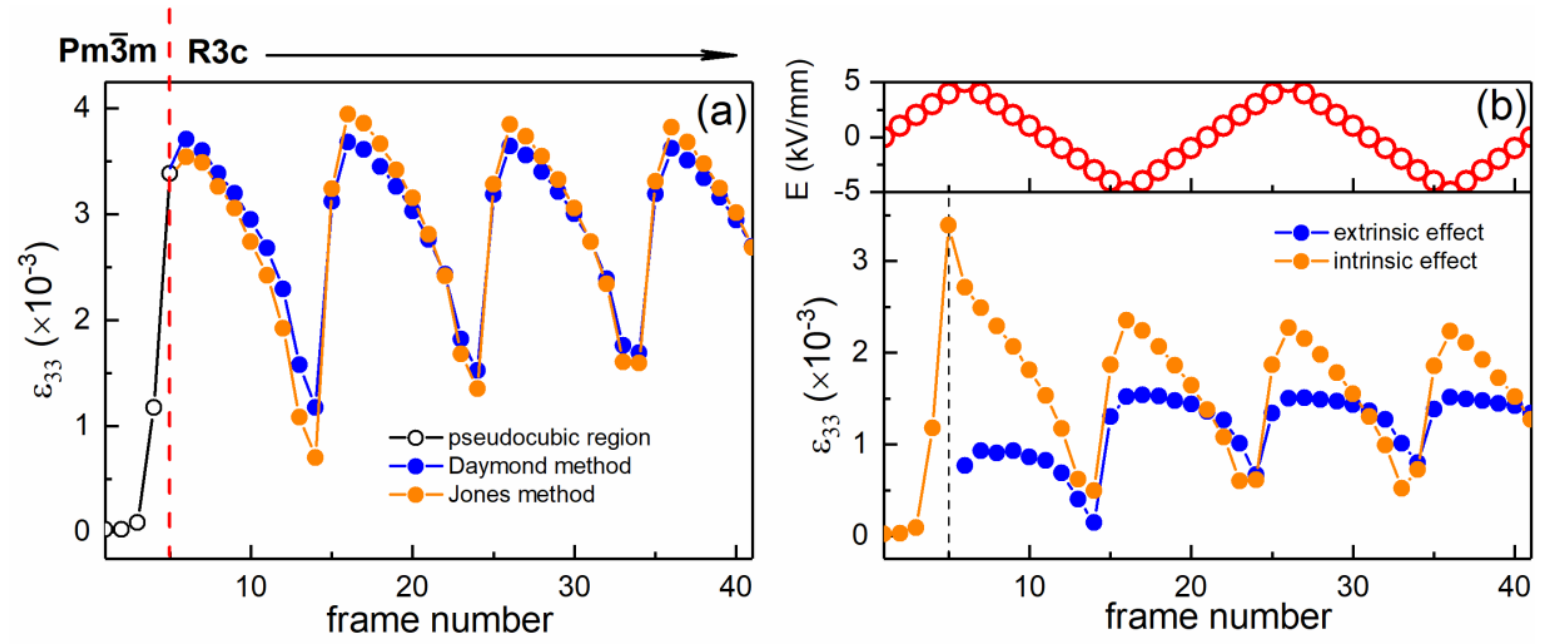

Figure 12. (a) Calculated macroscopic strain, $\varepsilon_{33}$, derived from two different methods, where the strain, $\varepsilon_{33}$, in the pseudocubic region was calculated according to the weighted-average lattice strain. (b) Variations in the intrinsic and extrinsic contributions to $\varepsilon_{33}$ with electric field based on the Jones method.

The S-E loop of $\varepsilon_{33}^{\text {total }}$, calculated according to the Daymond method is also illustrated in Figure $13(a)$, together with $\varepsilon_{11}^{\text {total }}$ and $\varepsilon_{v}$, which is consistent with the DIC measurement of macroscopic strain as shown in Figure 13 (b), the calculated and measured maximum $\varepsilon_{33}$ values being $3.75 \times 10^{-3}$ and $3.83 \times 10^{-3}$ respectively. The calculated S-E loops are not well defined because of the relatively large measurement step of $1 \mathrm{kV} / \mathrm{mm}$, which leads to a loss of resolution, especially around the coercive field. Furthermore, only small changes are observed in $\varepsilon_{v}$ due to the small difference between the unit cell volumes of the initial pseudocubic and electric field-induced rhombohedral phase, as listed in Table 1. 

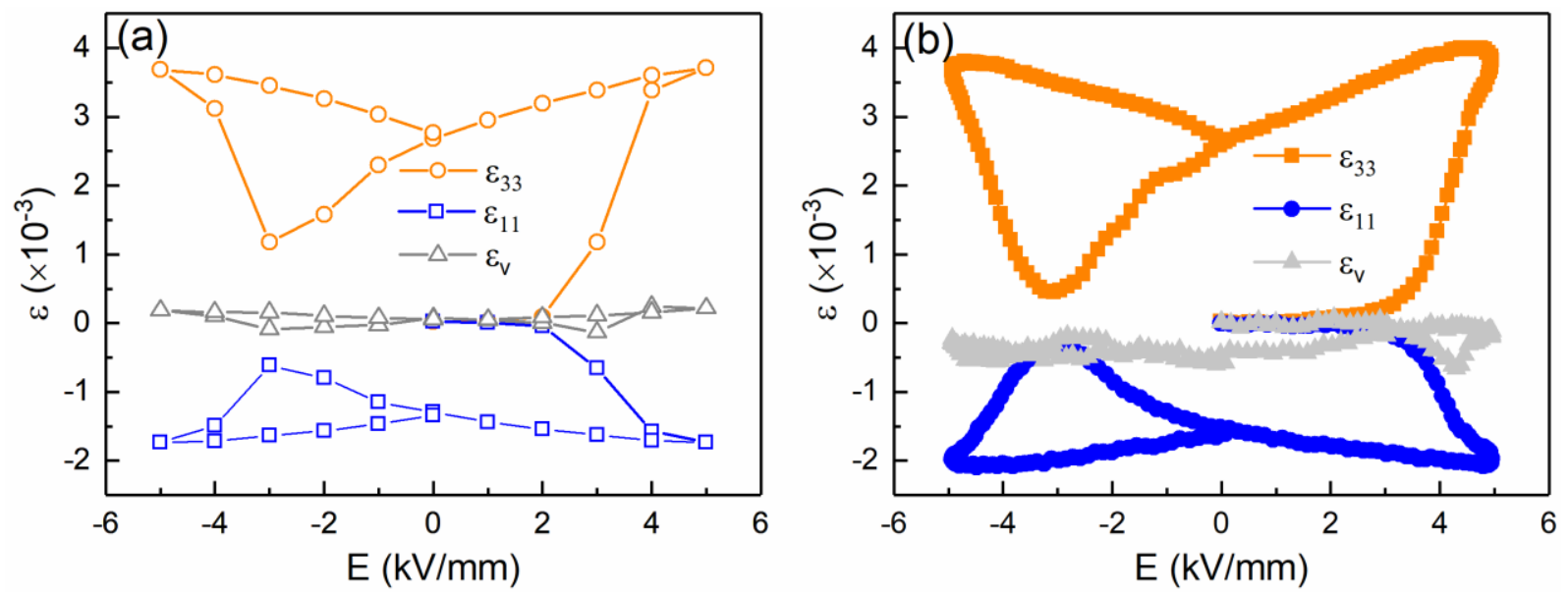

Figure 13. Macroscopic strain-electric field relationships determined from (a) analysis of XRD peak profiles using the Daymond method and (b) DIC measurements.

\subsection{Anisotropy analysis and elastic coupling}

The calculated effective lattice strains for $\{111\},\{200\}$ and $\{220\}$ at $\psi=0^{\circ}$ and $90^{\circ}$, determined according to Eq. 19, 20 and 21, are plotted in Figure 14. All the $\varepsilon_{\{h k l\}}$ values vary systematically with the frame number and correlate well with the variation in the applied electric field. $\varepsilon_{\{h k l\}}$ generally exhibits positive strain for $\psi=0^{\circ}$, while similar trends with opposite sign are found for $\psi=90^{\circ}$, in accordance with the expected lateral strain with opposite sign to longitudinal strain. It is also evident that the magnitude of longitudinal strain is almost twice that of the transverse strain for each grain orientation.

The values of $\varepsilon_{\{h k l\}}$ also depend on crystallographic orientation, with $\varepsilon_{\{200\}}$ exhibiting the largest variation among the tested grain families at any given electric field. However, the highest values of $\varepsilon_{\{111\}}, \varepsilon_{\{200\}}$ and $\varepsilon_{\{220\}}$ are still relatively close to each other, yielding $3.75 \times 10^{-3}, 4.02 \times 10^{-3}$ and $3.52 \times 10^{-3}$ for the first cycle respectively, which is much lower than the anisotropy previously reported in tetragonal soft PZT, where $\varepsilon_{\{200\}}$ was more than three times the value of $\varepsilon_{\{111\}}$ [21]. Furthermore, the highest effective lattice strains are expected to be $\varepsilon_{\{111\}}$ and $\varepsilon_{\{200\}}$ for rhombohedral and tetragonal phases respectively in normal ferroelectrics, such as soft PZT, where the extrinsic contribution dominates the 
electric field-induced macroscopic strain[11]. In the present study, both the intrinsic and extrinsic contributions play important roles in the macroscopic strain of the electric field-induced rhombohedral phase, and the distribution and configuration of intergranular stress should be distributed more evenly across differently-oriented grains due to the relatively low strain anisotropy.
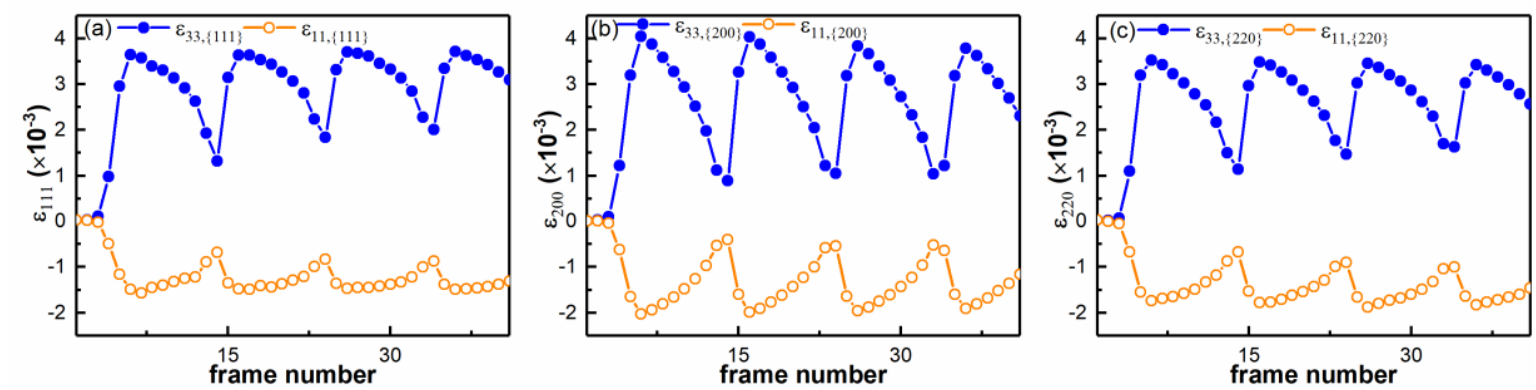

Figure 14. Variation in effective lattice strains $\varepsilon_{33}$ and $\varepsilon_{11}$ for (a) $\{111\}$, (b) $\{002\}$ and (c) $\{220\}$ grain families, illustrating relatively small strain anisotropy.

The observation of relatively low strain anisotropy seems to contradict the apparent discrepancy between the DODs of $<111>$ and $<220>$ poles, as shown in Figure 10 (c) and Figure 11 (a) i.e $M R D_{220}$ is approximately half that of $M R D_{111}$, even though the effective lattice strains, $\varepsilon_{\{111\}}$ and $\varepsilon_{\{200\}}$, are similar. It should be recognised that the MRD values for a given reflection incorporates both the domain switching fraction, $\Delta \eta$, and the multiplicity as indicated in Eq. 27. However, the domain switching fraction is the more pertinent factor for comparing the different degrees of domain switching, which were approximately 0.475 and 0.361 for $\Delta \eta_{111}$ and $\Delta \eta_{220}$ respectively. Furthermore, the contribution of the intrinsic effect (lattice strain) of differently-oriented grain families should also be considered. As shown in Figure 11 (b) and (c), the effective lattice strains, $\varepsilon_{\{111\}}$ and $\varepsilon_{\{200\}}$, were evaluated both with and without the intrinsic contributions; it is evident that the intrinsic contribution to $\varepsilon_{\{220\}}$ is greater than that for $\varepsilon_{\{111\}}$, due to the relatively large lattice strain for (220), with (111), (111) and $(\overline{2} 20)$ being relatively invariant. This effect is indicated by the evident shifts in the diffraction peaks as a function of the applied electric field and azimuthal angle, as illustrated in Figure 3 (f) andFigure 4 (f). 
The strain orientation distributions for $\{111\},\{200\}$ and $\{220\}$ were normalized by dividing by the corresponding maximum strain at $\psi=0^{\circ}$ for the remanent state and under $5 \mathrm{kV} / \mathrm{mm}$ respectively. As shown in Figure 15 (a) and (b), the absolute variations in the effective lattice strain show weak anisotropy as noted above. In the remanent (zero-field) state, $\varepsilon_{\{111\}}$ is dominant due to domain switching and the absence of the intrinsic piezoelectric effect in the poled state, while at $E_{\max } \varepsilon_{\{200\}}$ is largest as a result of the increased intrinsic contribution, shown previously in Figure 12 (b). The normalised SODs shown in Figure 15 (c) and (d) were almost identical to each other in both the remanent and maximum electric field states, which implies that the grain families of $\{111\},\{200\}$ and $\{220\}$ deformed to approximately the same degree as a function of the azimuthal angle, $\psi$, indicating the occurrence of strong elastic coupling between differently-oriented grains in order to minimize and accommodate the intergranular stresses.
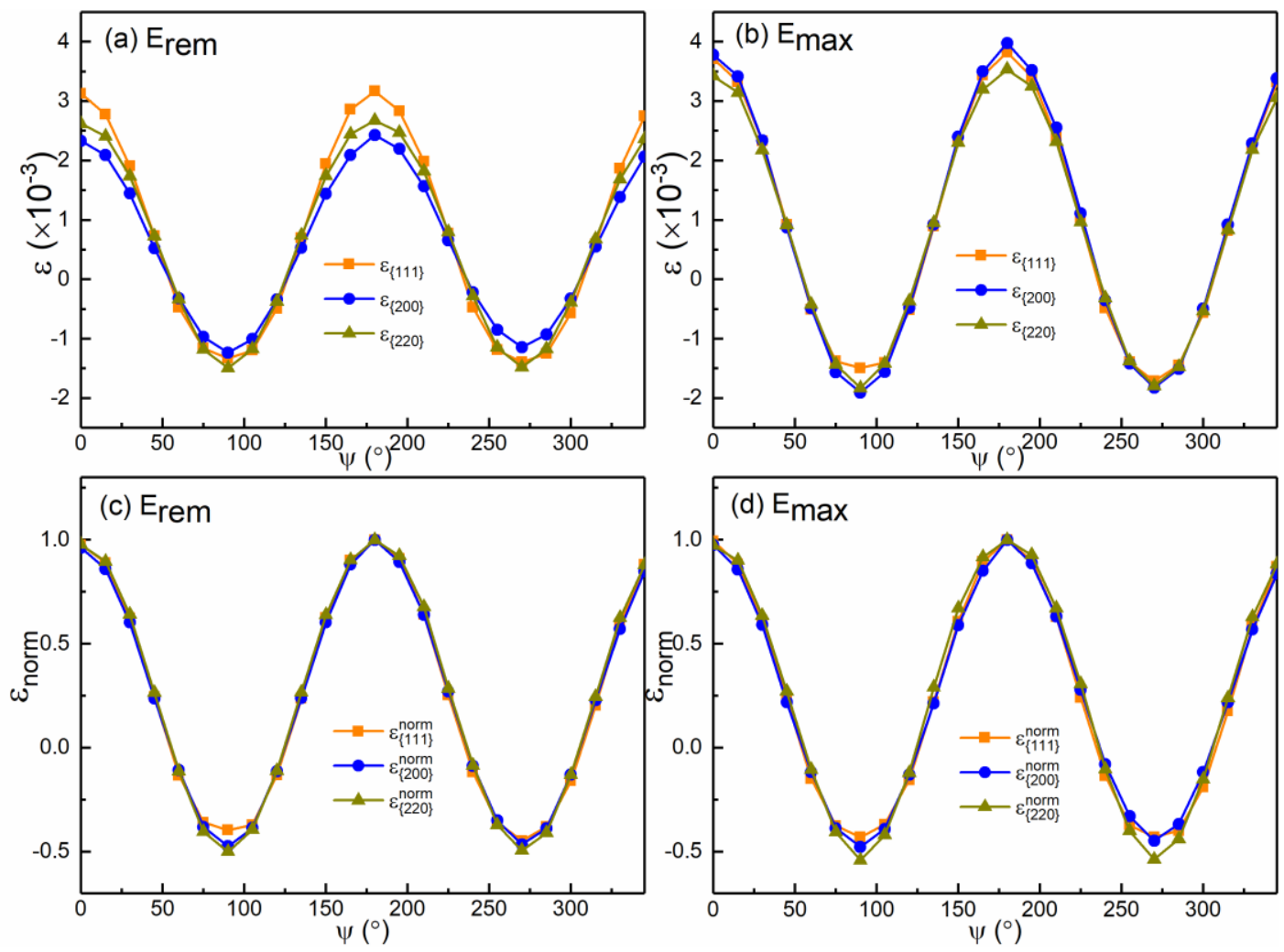

Figure 15. (a) and (b) the strain orientation distributions (SODs) for the $\{111\}$, $\{200\}$ and $\{220\}$ grain families in the remanent state and at $5 \mathrm{kV} / \mathrm{mm}$ respectively. (c) and (d) illustrated the corresponding normalized SODs for the $\{111\},\{200\}$ and $\{220\}$ grain families. 


\section{Conclusions}

In summary, an electric field-induced phase transformation was identified by an in-situ XRD experiment in the ceramic sample with composition $0.57 \mathrm{BiFeO}_{3}$ $0.21 \mathrm{~K}_{0.5} \mathrm{Bi}_{0.5} \mathrm{TiO}_{3}-0.22 \mathrm{PbTiO}_{3}$, which transformed from a NR pseudocubic to a FE rhombohedral phase. The XRD patterns for the transformation process were analyzed by methods including both single peak profile fitting and full pattern refinement, yielding good agreement. The invariant angle, $\psi_{0}$, was identified as $54.45^{\circ}$ by solving the intersection point of the fitted profiles for azimuthal angledependent $d_{(002)}$ values under various electric field levels; nonlinear fitting of the results was also conducted to determine the lattice spacings and peak intensity ratios of the randomly oriented, strain-free reference state. It is shown that fullpattern fitting of poled ceramic samples can be accomplished effectively by selecting the diffraction pattern corresponding to the invariant angle.

The microscopic strain-electric field response was revealed by evaluation of the effective lattice strains, together with intrinsic and extrinsic contributions. Relatively weak strain anisotropy was observed between the effective lattice strains of $\{111\},\{200\}$ and $\{220\}$ grain families $\left(3.75 \times 10^{-3}, 4.02 \times 10^{-3}\right.$ and $3.52 \times 10^{-3}$ at $5 \mathrm{kV} / \mathrm{mm}$ respectively), compared with the strong strain anisotropy reported previously for both rhombohedral and tetragonal normal ferroelectrics. According to the evaluation procedure of the intrinsic and extrinsic contributions to the macroscopic longitudinal strain, a dominant lattice strain (intrinsic effect) of $2.5 \times 10^{-3}$ was observed, compared with a strain of $1.5 \times 10^{-3}$ induced by domain switching (extrinsic effect) in the transformed rhombohedral phase, which contrasts with the usually dominant extrinsic strain contribution in normal ferroelectrics. These observations suggest the presence of lower-order local symmetries, which leads to enhanced polarization along different crystallographic directions besides $<111>$. Furthermore, strong elastic coupling was observed for the deformation of different grain families as a function of the azimuthal angle. In other words, all the grains deformed to a similar degree at a given azimuthal angle. The methodology developed in the present study has been successfully applied to evaluate the electric field-induced micromechanical behavior of the NR 
composition of BF-KBT-PT, and also has potential to be applied to the analysis of other ferroelectric perovskite solid solutions exhibiting a phase transformation from relaxor to single phase normal ferroelectric.

\section{Acknowledgements}

We thank Diamond Light Source for access to beamline I15 (proposal number EE16390-1) that contributed to the results presented here. 


\section{References}

[1] Z.G.G. Ye, Relaxor ferroelectric complex perovskites: Structure, properties and phase transitions, Key Eng. Mater. 155-156 (1998) 81-122. doi:10.4028/www.scientific.net/KEM.155-156.81.

[2] A.A. Bokov, Z.G. Ye, Recent progress in relaxor ferroelectrics with perovskite structure, J. Mater. Sci. 41 (2006) 31-52. doi:10.1007/s10853-005-5915-7.

[3] V. Bobnar, Z. Kutnjak, R. Pirc, A. Levstik, Electric-field-temperature phase diagram of the relaxor ferroelectric lanthanum-modified lead zirconate titanate, Phys. Rev. B. 60 (1999) 6420. doi:10.1103/PhysRevB.60.6420.

[4] A.A. Bokov, M. Maglione, Z.G. Ye, Quasi-ferroelectric state in $\mathrm{Ba}\left(\mathrm{Ti}_{1-\mathrm{x}} \mathrm{Zr} \mathrm{r}_{\mathrm{x}}\right) \mathrm{O}_{3}$ relaxor: Dielectric spectroscopy evidence, J. Phys. Condens. Matter. 19 (2007). doi:10.1088/0953-8984/19/9/092001.

[5] A. Kumar, I. Rivera, R.S. Katiyar, Investigation of local structure of lead-free relaxor $\mathrm{Ba}\left(\mathrm{Ti}_{0.70} \mathrm{Sn}_{0.30} \mathrm{O}_{3}\right.$ by Raman spectroscopy, J. Raman Spectrosc. 40 (2009) 459-462. doi:10.1002/jrs.2153.

[6] M. Acosta, W. Jo, J. Rödel, Temperature- and frequency-dependent properties of the $0.75 \mathrm{Bi}_{1 / 2} \mathrm{Na}_{1 / 2} \mathrm{TiO}_{3}-0.25 \mathrm{SrTiO}_{3}$ lead-free incipient piezoceramic, J. Am. Ceram. Soc. 97 (2014) 1937-1943. doi:10.1111/jace.12884.

[7] A. Zeb, S.J. Milne, Stability of High-Temperature Dielectric Properties for (1x) $\mathrm{Ba}_{0.8} \mathrm{Ca}_{0.2} \mathrm{TiO}_{3}-\mathrm{xBi}\left(\mathrm{Mg}_{0.5} \mathrm{Ti}_{0.5}\right) \mathrm{O}_{3}$ Ceramics, J. Am. Ceram. Soc. 96 (2013) 2887-2892. doi:10.1111/jace.12412.

[8] M.I. Morozov, M.A. Einarsrud, T. Grande, Polarization and strain response in $\mathrm{Bi}_{0.5} \mathrm{~K}_{0.5} \mathrm{TiO}_{3}-\mathrm{BiFeO}_{3}$ ceramics, Appl. Phys. Lett. 101 (2012) 252904. doi:10.1063/1.4772588.

[9] J. Rödel, K.G. Webber, R. Dittmer, W. Jo, M. Kimura, D. Damjanovic, Transferring lead-free piezoelectric ceramics into application, J. Eur. Ceram. Soc. 35 (2015) 1659-1681. doi:10.1016/j.jeurceramsoc.2014.12.013.

[10] J. Bennett, A. J. Bell, T.J. Stevenson, T.P. Comyn, Tailoring the structure and piezoelectric properties of $\mathrm{BiFeO}_{3}-\left(\mathrm{K}_{0.5} \mathrm{Bi}_{0.5}\right) \mathrm{TiO}_{3}-\mathrm{PbTiO}_{3}$ ceramics for high temperature applications, Appl. Phys. Lett. 103 (2013) 152901. doi:10.1063/1.4824652.

[11] A. Pramanick, D. Damjanovic, J.E. Daniels, J.C. Nino, J.L. Jones, Origins of electro-mechanical coupling in polycrystalline ferroelectrics during subcoercive electrical loading, J. Am. Ceram. Soc. 94 (2011) 293-309. doi:10.1111/j.1551-2916.2010.04240.x.

[12] D. A. Hall, A. Steuwer, B. Cherdhirunkorn, T. Mori, P.J. Withers, A high energy synchrotron $\mathrm{x}$-ray study of crystallographic texture and lattice strain in soft lead zirconate titanate ceramics, J. Appl. Phys. 96 (2004) 4245-4252. doi:10.1063/1.1787590. 
[13] J.L. Jones, M. Hoffman, K.J. Bowman, Saturated domain switching textures and strains in ferroelastic ceramics, J. Appl. Phys. 98 (2005) 0-6. doi: $10.1063 / 1.1988978$.

[14] M.C. Ehmke, N.H. Khansur, J.E. Daniels, J.E. Blendell, K.J. Bowman, Resolving structural contributions to the electric-field-induced strain in leadfree (1- $\mathrm{X}) \mathrm{Ba}\left(\mathrm{Zr}_{0.2} \mathrm{Ti}_{0.8}\right) \mathrm{O}_{3}-\mathrm{X}\left(\mathrm{Ba}_{0.7} \mathrm{Ca}_{0.3}\right) \mathrm{TiO}_{3}$ piezoceramics, Acta Mater. 66 (2014) 340-348. doi:10.1016/j.actamat.2013.11.021.

[15] J.E. Daniels, W. Jo, J. Rödel, V. Honkimäki, J.L. Jones, Electric-field-induced phase-change behavior in $\left(\mathrm{Bi}_{0.5} \mathrm{Na}_{0.5}\right) \mathrm{TiO}_{3}-\mathrm{BaTiO}_{3}-\left(\mathrm{K}_{0.5} \mathrm{Na}_{0.5}\right) \mathrm{NbO}_{3}: \quad \mathrm{A}$ combinatorial investigation, Acta Mater. 58 (2010) 2103-2111. doi:10.1016/j.actamat.2009.11.052.

[16] M. Hinterstein, M. Hoelzel, J. Rouquette, J. Haines, J. Glaum, H. Kungl, M. Hoffman, Interplay of strain mechanisms in morphotropic piezoceramics, Acta Mater. (2015). doi:10.1016/j.actamat.2015.04.017.

[17] N.H. Khansur, M. Hinterstein, Z. Wang, C. Groh, W. Jo, J.E. Daniels, Electricfield-induced strain contributions in morphotropic phase boundary composition of $\left(\mathrm{Bi}_{1 / 2} \mathrm{Na}_{1 / 2}\right) \mathrm{TiO}_{3}-\mathrm{BaTiO}_{3}$ during poling, Appl. Phys. Lett. 107 (2015) 0-5. doi:10.1063/1.4937470.

[18] A. J. Royles, A. J. Bell, J.E. Daniels, S.J. Milne, T.P. Comyn, Observation of a time-dependent structural phase transition in potassium sodium bismuth titanate, Appl. Phys. Lett. 98 (2011) 3-5. doi:10.1063/1.3582616.

[19] D. A. Hall, A. Steuwer, B. Cherdhirunkorn, T. Mori, P.J. Withers, Analysis of elastic strain and crystallographic texture in poled rhombohedral PZT ceramics, Acta Mater. 54 (2006) 3075-3083. doi:10.1016/j.actamat.2006.02.043.

[20] G. Wang, D.A. Hall, T.P. Comyn, L. Daniel, A.K. Kleppe, Structure and ferroelectric behaviour of $\mathrm{Na}_{0.5} \mathrm{Bi}_{0.5} \mathrm{TiO}_{3}-\mathrm{KNbO}_{3}$ ceramics, Adv. Appl. Ceram. 115 (2016) 89-95. doi:10.1080/17436753.2015.1104053.

[21] L. Daniel, D.A. Hall, J. Koruza, K.G. Webber, A. King, P.J. Withers, Revisiting the blocking force test on ferroelectric ceramics using high energy $\mathrm{x}$-ray diffraction, J. Appl. Phys. 117 (2015). doi:10.1063/1.4918928.

[22] J. Bennett, T.R. Shrout, S.J. Zhang, P. Mandal, a. J. Bell, T.J. Stevenson, T.P. Comyn, Temperature dependence of the intrinsic and extrinsic contributions in $\mathrm{BiFeO}_{3}-\left(\mathrm{K}_{0.5} \mathrm{Bi}_{0.5}\right) \mathrm{TiO}_{3}-\mathrm{PbTiO}_{3}$ piezoelectric ceramics, J. Appl. Phys. 116 (2014) 094102. doi:10.1063/1.4894443.

[23] J. Filik, A.W. Ashton, P.C.Y. Chang, P.A. Chater, S.J. Day, M. Drakopoulos, M.W. Gerring, M.L. Hart, O. V. Magdysyuk, S. Michalik, A. Smith, C.C. Tang, N.J. Terrill, M.T. Wharmby, H. Wilhelm, Processing two-dimensional X-ray diffraction and small-angle scattering data in DAWN 2, J. Appl. Crystallogr. 50 (2017) 959-966. doi:10.1107/S1600576717004708.

[24] Y. Li, Z. Zhang, Y. Chen, D.A. Hall, Electric field-induced strain in core-shell structured $\mathrm{BiFeO}_{3} \mathrm{~K}_{0.5}-\mathrm{Bi}_{0.5} \mathrm{TiO}_{3}-\mathrm{PbTiO}_{3}$ ceramics, Acta Mater. 160 (2018) 
199-210. doi:10.1016/J.ACTAMAT.2018.08.033.

[25] L. Lutterotti, S. Matthies, H.R. Wenk, A.S. Schultz, J.W. Richardson, Combined texture and structure analysis of deformed limestone from timeof-flight neutron diffraction spectra, J. Appl. Phys. 81 (1997) 594-600. doi:10.1063/1.364220.

[26] J.E. Daniels, W. Jo, J. Roedel, J.L. Jones, Electric-field-induced phase transformation at a lead-free morphotropic phase boundary. Case study in a 93\%( $\left.\mathrm{Bi}_{0.5} \mathrm{Na}_{0.5}\right) \mathrm{TiO}_{3}-7 \% \mathrm{BaTiO}_{3}$ piezoelectric ceramic, Appl. Phys. Lett. F. Full J. TitleApplied Phys. Lett. 95 (2009) 032904/1-032904/3. doi:10.1063/1.3182679.

[27] L. Daniel, D.A. Hall, P.J. Withers, A multiscale modelling analysis of the contribution of crystalline elastic anisotropy to intergranular stresses in ferroelectric materials, J. Phys. D. Appl. Phys. 47 (2014) 325303.

[28] W. A. Dollase, Correction of Intensities of Preferred Orientation in Powder Diffractometry: Application of the March Model., J. Appl. Crystallogr. 19 (1986) 267-272. doi:10.1107/S0021889886089458.

[29] N.C. Popa, D. Balzar, addenda and errata Elastic strain and stress determination by Rietveld refinement: generalized treat- ment for textured polycrystals for all Laue classes. Second corrigendum, J. Appl. Cryst. 47 (2014). doi:10.1107/S1600576714022109.

[30] H.-R. Kocks, U. F., Tomé, C. N., and Wenk, Texture and Anisotropy: Preferred Orientations in Polycrystals and their Effect on Materials Properties, 2nd ed, Cambridge University Press, Cambridge, 2000.

[31] Z. Wang, J.E. Daniels, Quantitative analysis of domain textures in ferroelectric ceramics from single high-energy synchrotron X-ray diffraction images, J. Appl. Phys. 121 (2017) 0-6. doi:10.1063/1.4982674.

[32] D.A. Hall, A. Steuwer, B. Cherdhirunkorn, P.J. Withers, T. Mori, Micromechanics of residual stress and texture development due to poling in polycrystalline ferroelectric ceramics, J. Mech. Phys. Solids. 53 (2005) 249260. doi:10.1016/j.jmps.2004.07.002.

[33] J.L. Jones, E.B. Slamovich, K.J. Bowman, Domain texture distributions in tetragonal lead zirconate titanate by $\mathrm{x}$-ray and neutron diffraction, J. Appl. Phys. 97 (2005). doi:10.1063/1.1849821.

[34] M.R. Daymond, The determination of a continuum mechanics equivalent elastic strain from the analysis of multiple diffraction peaks, J. Appl. Phys. 96 (2004) 4263-4272. doi:10.1063/1.1794896.

[35] BRUKER AXS GmbH, Karlsruhe, Diffrac Plus TOPAS v. 3.0 (Manual), 2006.

[36] V. Hauk, B. Krüger, Invariant intersections in diffraction stress analysis - a check of experimental results, Mater. Sci. Eng. A. 284 (2000) 261-267. doi:10.1016/S0921-5093(00)00729-2.

[37] C. Zhao, D. Hou, C.-C. Chung, H. Zhou, A. Kynast, E. Hennig, W. Liu, S. Li, 
J.L. Jones, Deconvolved intrinsic and extrinsic contributions to electrostrain in high performance, $\mathrm{Nb}$-doped $\mathrm{Pb}\left(\mathrm{Zr}_{\mathrm{x}} \mathrm{Ti}_{1-\mathrm{x}}\right) \mathrm{O}_{3}$ piezoceramics $(0.50 \leq \mathrm{x} \leq$ $0.56)$, Acta

Mater.158(2018)369380.doi:https://doi.org/10.1016/j.actamat.2018.08.006. 